\title{
Les biens culturels en Italie
}

De l'« assaut » à la mise en commun

\section{Daniela Festa}

\section{CpenEdition}

\section{Journals}

Édition électronique

URL : https://journals.openedition.org/insituarss/1148

DOI : 10.4000/insituarss. 1148

ISSN : 2680-4972

Éditeur

Ministère de la Culture

\section{Référence électronique}

Daniela Festa, « Les biens culturels en Italie », In Situ. Au regard des sciences sociales [En ligne], 2 |

2021, mis en ligne le 22 mars 2021, consulté le 23 janvier 2023. URL : http://journals.openedition.org/ insituarss/1148; DOI : https://doi.org/10.4000/insituarss.1148

Ce document a été généré automatiquement le 23 janvier 2023.

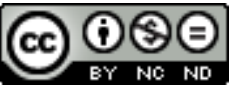

Creative Commons - Attribution - Pas d'Utilisation Commerciale - Pas de Modification 4.0 International - CC BY-NC-ND 4.0

https://creativecommons.org/licenses/by-nc-nd/4.0/ 


\title{
Les biens culturels en Italie
}

\author{
De l'« assaut ${ }^{1}$ » à la mise en commun
}

\section{Daniela Festa}

1 La prolifération de la littérature liée aux communs pousse à nous interroger sur la solidité de cette théorie émergente et sur sa capacité à améliorer les analyses et solutions formulées pour répondre aux questions de notre temps. Les biens culturels sont aujourd'hui de plus en plus associés aux biens communs ou parfois aux communs tout court. Néanmoins, ces références restent souvent floues et peu développées.

2 Pouvons-nous ainsi considérer les biens culturels en tant que biens communs ou communs? Peut-on interpréter la relation à ces biens sous l'angle de l'inclusivité ${ }^{2}$ et non pas de l'exclusivisme propriétaire et dans quel sens?

3 J'essaierai ici d'éclaircir le lien entre ces notions à partir de la récente élaboration italienne. Les arguments qui suivront, bien que porteurs d'une dimension paradigmatique, sont toutefois doublement situés : ils sont, d'une part étroitement liés à l'accélération des politiques néolibérales mises en œuvre ces dernières décennies en Italie et plus largement en Europe, et demeurent d'autre part internes au débat italien sur la dimension collective des droits. Cette question connait ici une trajectoire propre influencée par une approche particulière du patrimoine territorial et incarnée dans des notions juridiques spécifiques telles qu'une propriété fonctionnalisée appuyée sur les interprétations constitutionnelles.

4 Cette précaution posée, je considère néanmoins qu'aborder le patrimoine culturel sous le prisme du binôme bien communs / communs représente, de manière plus générale, une perspective privilégiée pour mettre en évidence les spécificités conceptuelles et les imbrications territoriales et politiques entre ces deux notions. 


\section{Unité patrimoniale et pluralisme propriétaire}

\section{Quelques références concernant propriété et communauté dans la Constitution}

5 Avant d'approfondir la liaison entre patrimoine culturel et communs à partir des pistes défrichées en Italie, rappelons en préambule quelques repères fondamentaux sur la notion de propriété au sein de cette tradition juridique. En premier lieu, il nous faut considérer une particularité du système constitutionnel d'aujourd'hui née de la réaction unitaire des forces démocratiques du pays à l'horreur de l'expérience fasciste. Dans le cadre de la Constitution de 1948, le droit de propriété ne compte pas parmi les principes fondamentaux mais figure au sein du titre consacré aux rapports socioéconomiques, et est par conséquent subordonné à ces principes. L'art. 42 de la Constitution soumet ce droit à ladite fonction sociale de la propriétée ${ }^{3}$. Cette formule, déjà notoirement mobilisée dans la pensée juridique continentale ${ }^{4}$ et évoquée auparavant dans la Constitution de Weimar (1919), trouve toutefois ici une consécration explicite et renvoie au large débat qui avait animé les deux rives de l'océan Atlantique entre la fin du XIX et le début du Xxe siècle ${ }^{5}$.

6 De fait, ni la collocation dans le titre de la Constitution italienne consacré aux rapports économiques, ni la référence à cette prééminente fonction n'auront affaibli le «terrible droit » (Rodotà 2013) héritier de la tradition bourgeoise post-révolutionnaire française et globalement érigé en un droit cardinal tout au long de la modernité. Dans les années qui suivront l'approbation de la Charte constitutionnelle, une interprétation compromissoire prédominera dans les faits afin d'articuler cette norme progressiste avec l'expansion d'un capitalisme de masse. Néanmoins, cette déclinaison d'une propriété attentive aux besoins sociaux, qui peut s'inscrire dans le cadre de la pensée sociale ${ }^{6}$ qui influencera globalement les constitutions de deuxième génération, aura su offrir, à différentes reprises, un ancrage à des argumentations juridiques orientées vers une vision redistributive du droit.

7 Il est d'autre part important de relever que la notion d'accès est contenue dans cette norme de la fonction sociale de la propriété7. Le mot semble entériner ici l'expression iconique «pas tous prolétaires mais tous propriétaires» pour caractériser le compromis entre les forces communistes et les chrétiens modérés au sein de l'Assemblée constituante. Cette "révolution promesse " a constitué à l'inverse un simple engagement programmatique promouvant une extension progressive de l'accès à la propriété

8 Toutefois, en vertu du caractère abstrait du droit qui lui permet d'être réinventé et détourné par des projets politiques disparates et parfois opposés, cette référence à l'accès à la propriété composera finalement l'un des pivots d'ancrage constitutionnel de la théorie des communs. Nous verrons par la suite que l'accès devient la clef d'un nouveau projet social dans la lecture progressiste de Stefano Rodotà, vision d'ailleurs reprise par la Cour de cassation. L'accès promis et promu ne serait pas dans ce cas celui de la titularité mais celui des utilités qu'un bien "indépendamment de sa titularité » peut fournir. L'angle sous lequel sont observés les biens dans le but de garantir l'épanouissement de la personne n'est plus celui du dominium construit comme le hendiadys de la liberté, mais celui de l'accès effectif aux utilités que les biens peuvent socialement réaliser. 
9 La norme qui suit (art. 43 de la Constitution ${ }^{9}$ ), elle aussi largement réinterrogée par le mouvement des communs, admet la possibilité (en cas d'un intérêt général prééminent) de transférer des entreprises de services publics essentielles à « des communautés de travailleurs ou d'usagers ". On reviendra sur l'activation de cette norme, en outre quasiment oubliée. Nous pouvons anticiper d'ores et déjà que bien que la fonction primaire de cet article fût d'admettre la nationalisation de certains services essentiels en réservant uniquement à la loi une telle initiative, Salvatore Pugilatti, civiliste éminent, soulignait déjà la portée novatrice de la norme (Pugliatti 1964 : 224). Selon le juriste, elle offrirait une vision renouvelée de la dimension collective de la propriété. En marge des propriétés et des droits fonciers collectifs en milieu rural et montagnard, cette norme proposerait que des groupes de travailleurs et d'utilisateurs puissent assumer une importance cruciale dans d'autres secteurs de l'économie du pays. Les constituants visaient probablement les formes coopératives largement amorcées dans la Péninsule déjà à l'époque pré-unitaire, toutefois ils ont opté pour une formule bien plus large, ce qui ne peut être considéré comme anecdotique. En évoquant la simple « communauté de travailleurs ou d'usagers » la norme admet, en principe, le transfert de ces services essentiels non uti singuli mais sous la forme génériquement communautaire à des collectivités même hors de toutes personnifications et formalisation juridiques. 
Figure 1

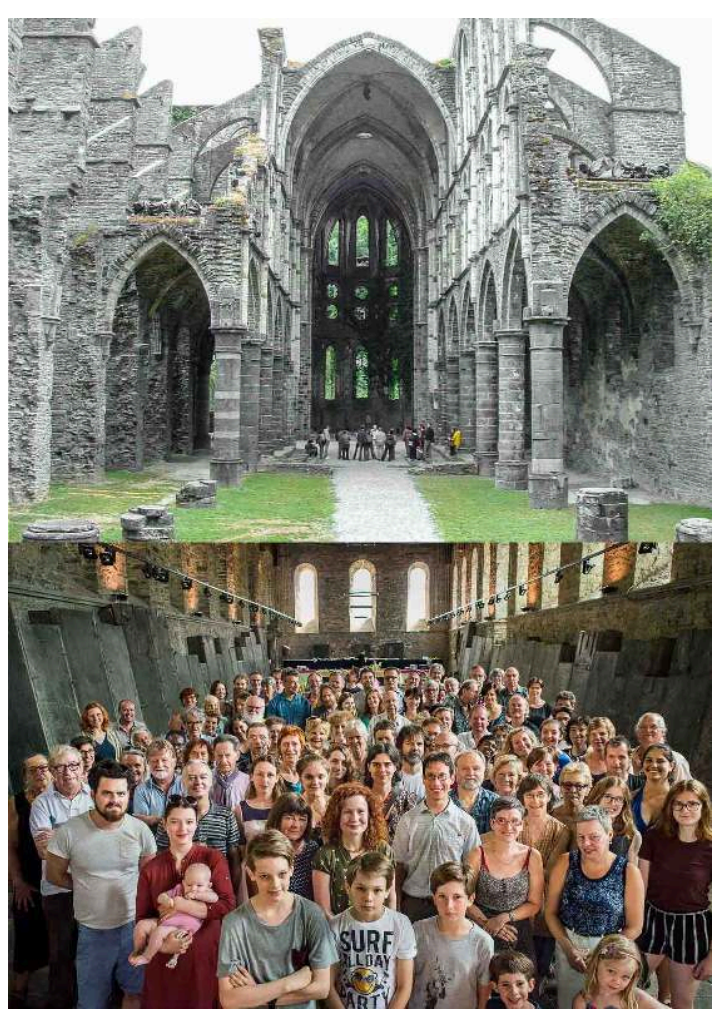

La large communauté Buren van de Abdij ( "Voisins de l'abbaye ») est un groupe de contributeurs aux centres d'intérêt variés qui organisent des évènements et des visites guidées, et participent aux groupes de travail dédiés à la gestion de l'abbaye Saint-Bavon (Gand, Belgique). Cette abbaye fondée au milieu du vıle siècle fut active jusqu'en 1539 jusqu'à ce que, en représailles contre les Gantois révoltés, Charles Quint décide de la transformer en une citadelle militaire reliée aux remparts de la ville. Par la suite, l'histoire des bâtiments de l'abbaye est restée liée à l'histoire militaire de la ville de Gand. Au xvi siècle, les troubles religieux affectèrent l'ancienne abbaye, qui partiellement détruite par les calvinistes (1581). Le site resta ensuite peu connu et peu accessible jusqu'à sa prise en charge directe par les Voisins de l'abbaye.

EN HAUT $\odot$ G. Debognies, distr. Creative Commons (licence CC BY-SA 3.0) ; EN BAS @ Sammy Van Cauteren.

\section{L'unité du patrimoine : la prospective territoriale}

Concernant plus particulièrement le patrimoine culturel, la possibilité de limiter l'exclusivisme propriétaire sur un bien culturel ${ }^{10}$ est en cohérence avec cette vision d'une propriété socialement fonctionnalisée, mais trouve ses fondements dans l'art. 9 de la Constitution ${ }^{11}$.

11 La norme, située quant à elle parmi les principes fondamentaux de la Constitution, affirme: «La République favorise le développement de la culture et la recherche scientifique et technique. Elle protège le paysage et le patrimoine historique et artistique de la Nation. » La constitutionnalisation de la protection du patrimoine est un autre trait spécifique des constitutions $d u x^{e}$ siècle qui ne se limitent plus à organiser les pouvoirs et à fixer un noyau essentiel de principes, mais développent un programme et en détaillent les orientations. La notion de patrimoine, distincte de celle que développe le droit commun, évoque un héritage reçu des générations précédentes à transmettre aux générations futures. Cette formulation particulière associant nature et culture trouve dans la Constitution weimarienne (art. 150) son antécédent tandis que 
déjà la Constitution républicaine espagnole (1931) reconnaissait la titularité de la nation selon une formulation élaborée en France. En effet et à l'époque révolutionnaire, les biens confisqués à l'Église, puis à la noblesse et ceux de la Couronne, acquièrent le statut de «biens nationaux » et, selon la fictio de la personnalité morale, forment le patrimoine de la nation qui assume la responsabilité de sélectionner ce qui doit être transmis aux générations futures. L'urgence était également de limiter le saccage systématique d'œuvres et de monuments emblématiques de l'ordre social précédent, puis de reformuler une identité collective. Patrimoine et paysage, comme dans l'approche française, "appartiennent» donc à la nation tandis que la République Italienne est le sujet responsable de leur protection. Plus récemment avec le Codice dei bieni culturali et del paesaggio (2004), cette titularité se clarifie. Le Code, en suivant l'exemple des sources internationales (voir l'approche de la convention Unesco de 2003 sur les biens immatériels confirmée ensuite par la convention de Faro de 2005) rattache le patrimoine culturel et paysager à «la communauté nationale» en soulignant davantage la dimension anthropique et culturelle plutôt que la signification organique ou institutionnelle de cette référence.

12 À l'époque de son élaboration, le propos principal de l'art. 9 était de soustraire le secteur patrimonial à la contingence politique pour en faire un pilier des engagements républicains. La norme représente par ailleurs un avancement considérable dans la vision du patrimoine culturel jusque-là plutôt élitiste et vouée à la seule conservation (Bartolini 2013). Patrimoine et culture se trouvent ainsi strictement associés et interdépendants dans une vision largement plus dynamique. Le patrimoine historique et artistique est de prime abord et de manière originale associé au paysage, et par ailleurs, les deux sont strictement liés au développement de la culture et de la recherche : dans la volonté de l'Assemblée constituante, leur protection représente de fait un pas incontournable vers un plus large développement culturel et scientifique, préoccupation primaire de la norme (Sandulli 1967, II : 69, 70).

Cette connexion entre patrimoine et paysage - et donc, via ce dernier, avec le territoire en tant que processus qui se représente à travers un paysage - constitue un trait distinctif de la tradition italienne. Bien que les disciplines juridiques des biens culturels et des biens paysagers soient distinctes au sein du Code qui leur est conjointement consacré - ce qui affaiblit considérablement leur caractère unitaire - leur connexion demeure très significative à différents égards.

14 La décision de laisser les biens artistiques et archéologiques dans les territoires d'origine fut par exemple le résultat d'une longue querelle déjà au sein de l'État de Savoie quand enfin la décision de valoriser l'ancrage territorial de ces biens prévaudra sur le modèle de musée pédagogique (qui aurait signifié le transfert d'une grosse partie des trésors artistiques et archéologiques du Sud dans la capitale du royaume) (Settis 2002:107 [version e-book]). La capillarité et la diffusion du patrimoine culturel sera le résultat de cette tradition dans toute la Péninsule et représente aujourd'hui une des composantes de la richesse du paysage culturel italien.

La connexion des biens culturels et des biens paysagers trouve ensuite sa source dans la pensée géographique et dans les études urbaines qui ont influencé le Code et plus largement l'approche de l'aménagement. Une école territorialiste originale ${ }^{12}$ a ainsi développé cette vision intégrée à partir de travaux critiques réalisés dans les années 1970 par des intellectuels tels que Lucio Gambi $^{13}$ et Antonio Cederna (2013) contre la spéculation immobilière et pour la sauvegarde du patrimoine territorial. Grâce à cet 
héritage, la dichotomie entre nature, culture et une vision esthétique et élitiste du patrimoine culturel est aujourd'hui globalement dépassée. La distinction entre patrimoine culturel et patrimoine paysager a perdu progressivement sa raison d'être pour laisser la place à une vision historicisée et politique de processus territoriaux unitaires qui touche à un milieu désormais largement anthropisé où une notion puriste de la nature demeure désormais abstraite. Même lorsqu'on parle de haute montagne, là où l'homme est presque absent dans la reproduction de cycles naturels, le paysage est néanmoins lié à l'importance que l'homme accorde à ces lieux, à leurs fonctions matérielles et symboliques et à leur protection en les transformant dans des «occurrences émotionnelles ${ }^{14}$ ».

La ligne de pensée mettant au centre le territoire comme processus coévolutif unitaire entre une société et son milieu naturel et culturel (Magnaghi 2014) marque le caractère collectivement produit du territoire et de ses configurations paysagères et culturelles. Nous retrouvons l'écho de cette vision dans le Code de 2004 (art. 2.3) qui reprend la même approche de la Convention européenne du paysage (2000) où le terme « paysage » désigne une partie de territoire telle qu'elle est perçue par les populations, dont le caractère résulte de l'« action de facteurs naturels et/ou humains et de leurs interrelations ».

17 Il faut par ailleurs observer l'oubli de l'art. 9 suite à l'approbation de la Constitution, auquel contribua largement l'émergence de la question environnementale et paysagère consécutive aux dévastations d'une expansion industrielle et immobilière rampantes. À partir des années 1970, l'importante saison de revendications environnementales invitera doctrine et jurisprudence à réinterroger et revitaliser cette norme. L'art. 9 se trouve aujourd'hui réécrit par les nombreuses décisions, devenant ainsi la pierre angulaire de la question territoriale, tant comme principe fondateur de cette matière que plus particulièrement concernant la santé publique et la protection de l'environnement (conjointement aux art. 2, 32, 41 de la Constitution).

Ce rapprochement avec le paysage a ainsi contribué à éclaircir la composante immanente et vivante du patrimoine, non seulement comme héritage du passé mais comme ensemble d'éléments constamment réappropriés et potentiellement sources de nouvelles valeurs sociales. Toujours au sujet des hybridations et évolutions subies par la notion de patrimoine culturel, nous devons constater que plus récemment le tournant du patrimoine immatériel ${ }^{15}$ a exercé une tension ultérieure sur la conception des biens culturels en mettant davantage en évidence la contribution créative produite par une communauté lorsqu'elle se réapproprie des valeurs culturelles.

\section{Jurisprudence des biens communs}

19 Pour confirmer une fois de plus la centralité du dépassement de la dichotomie natureculture dans cette matière (Marella 2016), il faut signaler l'importance majeure de la décision de la Cour de cassation de $2011^{16}$ qui se situe dans un ensemble cohérent de décisions de la même période et qui s'articule autour de la notion de paysage pour parvenir à revisiter la théorie des biens dans le système italien. Cette décision influencera ensuite largement la réflexion sur les biens communs en Italie et les revendications au sujet du patrimoine culturel. Dans le cadre d'un jugement notoire portant sur la titularité d'une des vallées de pêche de la lagune de Venise (Valle Averto), les Sections réunies de la Cour de cassation développent un argumentaire 
organique au sujet des biens, de leurs fonctions et, en conséquence de ces dernières, de leurs régimes (Napoli 2014: 211-233). La Cour déclare ainsi que la systématisation juridique des biens doit être considérée dans son ensemble (p. 8), la seule classification civiliste (déjà objet de nombreuses critiques par le passé) ne pouvant plus suffire (p. 11) à régler une matière aussi cruciale pour réaliser le projet constitutionnel de " protection de la personne humaine et de son développement au sein de l'état social » (p. 13). La Cour de cassation conclut: «Au-delà de la titularité formelle, il y a des biens (par exemple) de type environnemental et paysager [...] qui sont à considérer communs au-delà de la perspective désormais datée du dominium romain et de la propriété civile, c'est à dire affectés aux intérêts de tous les citoyens"(p.13) ${ }^{17}$. Elle appuie ses arguments sur la lecture combinée de l'art. 9 (protection du patrimoine et du paysage) et de l'art. 42 de la Constitution (propriété et fonction sociale), du principe fondamental de l'art. 2 de la Constitution (garantie constitutionnelle des droits fondamentaux de l'individu et «des formations sociales où il développe sa personnalité ») et sur leur "directe applicabilité ». En résulte une prééminence de l'affectation par rapport à toutes éventuelles titularités quand les biens en question sont indispensables pour réaliser " des intérêts fondamentaux pour le développement de la personne humaine " (p.23) selon la perspective personnaliste située au cœur de la construction constitutionnelle par l'art. 2 de la Constitution. La référence aux droits fondamentaux représente ici une claire allusion aux conclusions de la Commission Rodotà ${ }^{18}$ qui avait ainsi indiqué le critère d'identification des biens communs.

La décision propose un argumentaire riche et évoque d'autres exemples de disjonction entre titularité et jouissance dans le droit positif. La Cour cite à ce propos les droits d'usages publics, les droits fonciers collectifs et les propriétés collectives. Dans tous ces cas, la titularité formelle perd sa centralité face aux intérêts collectifs réalisés par une distribution des utilités rattachables aux biens. Nous sommes au cœur d'une vision pluraliste et fonctionnaliste de la propriété. Dans la tradition juridique italienne, de Pugliatti à Giannini et de Paolo Grossi à la lecture la plus progressiste de Stefano Rodotà, la propriété ne constitue pas un modèle unitaire strictement dépendant du paradigme exclusif. La décision se rapporte alors à cette ligne interprétative qui, bien que minoritaire dans le cadre des politiques des dernières décennies, fait désormais référence pour les juges de la Cour suprême. 
Figure 2

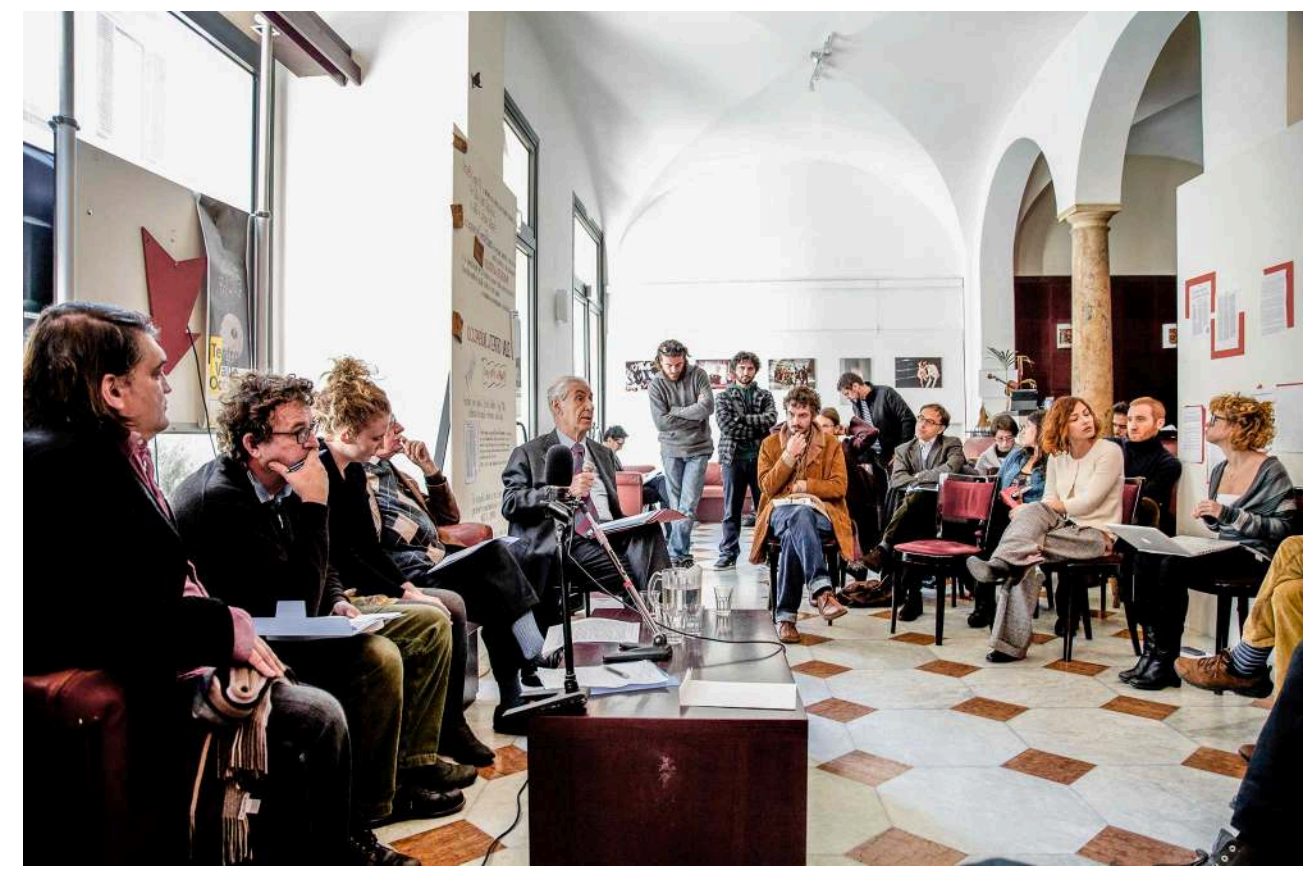

Stefano Rodotà, doyen des biens communs, dans une de ses nombreuses interventions au Teatro Valle Occupato. Ici, Rodotà se trouve parmi les activistes et les théoriciens des communs réunis dans le foyer du théâtre pour la journée de lancement de la Constituante des biens communs (13 avril 2013), projet extra-institutionnel de reprise et de mise à jour du texte élaboré auparavant par la commission ministérielle (dite Commissione Rodotà) présidée par l'éminent juriste.

(c) Valeria Tomasulo.

Précisons enfin que même si la Cour mentionne les caractères intrinsèques d'un bien, ses arguments ne sont jamais essentialistes. Ainsi, il ne s'agit pas d'inférer un régime à partir du «seul élément physique et morphologique » mais de poser au centre des considérations juridiques le lien entre ces caractéristiques et les fonctions qui peuvent être socialement développées à partir de celui-ci, rappelle explicitement la Cour parlant d'une prééminente "signification fonctionnelle de la domanialité » (p. 24). Dans cette décision et autant que dans l'élaboration de la Commission Rodotà qui constitue la référence doctrinale sous-jacente, ce procédé juridique évite l'impasse de toutes considérations essentialistes. Les fonctions et les utilités développées dans l'interaction entre l'homme et un tel objet spécifique sont les clefs interprétatives pour inférer son régime, non la naturalité ou la nature du bien. Ainsi, les fonctions territoriales et paysagères que le bras de mer salée en contentieux (techniquement aménagé en écluses) peut développer restent au cœur des préoccupations de la Cour. De fait, considérer le bien exclusivement comme une portion de mer importe moins. La notion de paysage y est, elle aussi, dépouillée de toutes qualifications esthétiques : la Cour fait directement référence aux usages de pêche et de navigation, aux usages du territoire pleinement productifs et qui le configurent en tant que bassin de pêche.

\section{Propriété divise et pluralisme propriétaire}

Concernant plus particulièrement le régime des biens culturels, lorsque ces biens appartiennent à l'État, l'utilisation collective est garantie sous la forme d'un service public respectueux de leurs conditions matérielles et de leur protection. Pour le 
patrimoine culturel privé, les limitations des pouvoirs propriétaires trouvent leur justification dans l'intérêt collectif à la protection, voire à la jouissance du bien. Cependant, il n'existe aucun principe d'accessibilité et d'utilisation générale, même si l'on en trouve des exemples significatifs dans le Code des biens culturels et du paysage de 2004 (Code BCP). En premier lieu, le dicatio ad patriam et le deputatio ad cultum, caractérisés par la mise à la disposition du public pendant une période prolongée du bien culturel (art. 105 du Code BCP) : dans ce cas, le droit reconnait un droit d'usage public (et non pas une servitude publique comme on l'affirme parfois) qui implique une accessibilité publique à perpétuité du bien. Enfin, les biens culturels privés qui ont fait l'objet de subventions publiques doivent être destinés à un usage collectif selon des méthodes établies au cas par cas (art. 38 du Code BCP). L'attribution de la qualité de bien culturel à la chose laisse ainsi inaltéré le droit du propriétaire, mais le transforme dans son contenu sans pourtant décréter le passage en propriété publique ou sa transformation ipso iure en bien collectif. Le ius excludendi sera en fait limité en vue de la protection et de la jouissance collective, le ius abutendi sera quant à lui exclu ${ }^{19}$. Mais comment alors interpréter cette figure si particulière de propriété ?

Si la Constitution italienne adopte la formule « patrimoine historique et artistique de la nation", cet ensemble de choses était autrefois désigné différemment. Le premier véritable objet juridique au sens patrimonial fut les «mémoires ${ }^{20}$ ». Plus tard, l'État pontifical publia en 1820 l'édit concernant les antiquités et les excavations. Ce n'est que dans la période post-unitaire que le législateur adoptera, d'abord avec la loi Rosadi de 1909 et ensuite avec la loi Bottai de 1939, la formule «objets d'art » qui constitue l'antécédent des biens culturels d'aujourd'hui. Ces lois qui avaient encore une approche plutôt traditionnelle assuraient la tutelle des objets d'art à travers des mesures essentiellement conservatoires et grâce à un régime de police administrative qui veillait à l'intégrité des objets classés.

Dans un premier temps, des interprétations destinées à sauvegarder l'unité du schéma propriétaire furent proposées afin de justifier la particularité du régime par de seules limitations administratives. Toutefois, cette option fut bientôt dépassée faute d'être capable d'expliquer l'engagement actif du public à l'égard de ces biens. Une conception fonctionnelle de la propriété fut alors avancée à partir de la lecture de ces lois pour tenir compte de la coprésence de la propriété et de l'intérêt social de sa protection et de sa jouissance. C'est donc autour des objets d'art que l'approche fonctionnaliste de la propriété s'est forgée. Il s'agit de la même ligne interprétative qui nous conduit aujourd'hui à concevoir certains biens comme communs ${ }^{21}$. Ils s'opposent à la propriété exclusive non pas parce qu'ils sont incompatibles avec tous titres d'appartenance, comme le voulait une première classification essentialiste, mais parce que c'est la fonction de tels biens qui détermine leur régime. À l'époque de ces premières lois, la doctrine parlait déjà de deux pouvoirs sur le même bien : «Un pouvoir public de protection et un pouvoir privé d'appartenance. » Ces deux droits sont simultanément garantis par le système juridique, "l'un étant fonctionnel, l'autre ayant un caractère privé». Le droit public est donc en "coexistence avec la relation propriétaire» (Grisolia 1952 : 44-45).

25 La théorie de la propriété accompagnant la loi Rosadi se fondait sur l'idée que la propriété d'œuvres d'art est une propriété sui generis. Elle sera reprise entièrement par Grisolia dans l'élaboration de la loi Bottai et systématisée ensuite par Massimo Severo Giannini, père fondateur du droit administratif et grand spécialiste des biens. Si la 
titularité du bien peut appartenir à une personne privée ou à une institution publique, sa jouissance, précise Giannini, appartient à « un ensemble d'usagers, c'est-à-dire à un groupe informel de personnes physiques, indéterminées et indéterminables [...] identifiables concrètement dans des groupes particuliers ${ }^{22}$ ", en conséquence de quoi les biens sont à considérer comme publics (Giannini 1976 : 13) au-delà de leur titularité. Cet argument montre bien une fois de plus la ligne doctrinale reprise par la Commission Rodotà et confirmée ensuite par la Cour de cassation ${ }^{23}$.

Dans la taxinomie de Giannini, la propriété peut être, du fait de sa structure: individuelle, collective ou divise. Les objets d'art représenteraient ainsi un exemple typique de propriété divise. Celle-ci n'est pas le contraire de la propriété indivise mais, pour utiliser les mots de Wesley N. Hohfeld ${ }^{24}$, un démembrement du bundle of right entre plusieurs sujets. Dans ce type de propriété, l'élément de l'appartenance se trouve réparti au regard de deux intérêts différents simultanément protégés par la norme à travers l'attribution des utilités rattachées à la chose à deux sujets distincts. L'objet d'art serait le support de plusieurs utilités incluant celles appartenant au titulaire (public ou privé), et celles inhérentes à la valeur culturelle immatérielle incorporée dans le bien qui est toujours « publique ${ }^{25}$ » ou selon la terminologie la plus récente de la Cassation, «commune » et donc domaniale dans le sens «collectif et fonctionnel» du terme.

Une conception semblablement dualiste avait déjà été évoquée dans la pensée extrajuridique. «Il y a deux choses dans un édifice : son usage et sa beauté. Son usage appartient au propriétaire, sa beauté à tout le monde ", proclamait notoirement Victor Hugo dans ses écrits à propos de la destruction des monuments en France (Hugo 1832). Mais à la différence de l'Italie, cette idée de désarticulation entre titularité et jouissance reste globalement étrangère à la pensée juridique française ${ }^{26}$ qui considère majoritairement la propriété divisée comme une réminiscence de l'Ancien Régime (Rochfeld à paraitre) et qu'elle est incompatible avec le droit de propriété moderne. Pourtant, une doctrine illustre construit la figure de la transpropriation au sujet de biens environnementaux de façon assez comparable, bien qu'àtravers la figure du patrimoine. Ainsi, c'est en passant par la notion hybride et complexe de patrimoine commun de la nation ou de l'humanité que François Ost (1995a: 306 sq) justifie la concession d'usages multiples à une diversité de titulaires pour réaliser une forme réussie de jouissance patrimoniale collective (Ost 1995b : 102). Ce patrimoine, de façon similaire au patrimoine de la tradition civiliste, apparaît alors lui aussi comme « un ensemble de biens dans le commerce et d'éléments immatériels renvoyant à l'identité de leur titulaire ${ }^{27} »$. Il présente toutefois des traits particuliers. Il s'agit ainsi d'un patrimoine collectif impliquant qu'une " protection recherchée procède donc autant du souci de préservation d'un capital que de la volonté de sauvegarder des valeurs constitutives de l'identité du groupe». De plus et à l'égard de ces biens environnementaux placés sous la protection d'un patrimoine collectif, « le propriétaire ou le souverain sera tenu d'agir comme un mandataire responsable et désormais comptable de leur gestion ».

Cette figure regagne de l'intérêt parmi les théoriciens des communs qui en reprennent la structure sous l'angle de la destination, voire de l'affectation de certains biens. La disjonction entre le sujet titulaire et le sujet affectataire n'est pas nouvelle en matière de biens culturels. Le droit de propriété en France, s'exerçant sur ces choses, note Marie Cornu (2005 : 1452-1453), est soumis à des limitations et intrusions depuis la loi 
de 1913 au nom de l'intérêt collectif de la nation. Le fait qu'un bien soit approprié n'empêcherait pas de le considérer comme destiné, dans ce cas, on constaterait une propriété compatible avec les intérêts de tiers à utiliser ou préserver certaines de ses utilités, observe Judith Rochfeld (2017 : 244), en adaptant de façon originale le terme de transpropriation et en mettant en évidence, de manière similaire à celle de Massimo Severo Giannini, le caractère potentiellement diffus et non nécessairement déterminé de la communauté bénéficiaire. Comme le mentionne Giannini dans sa construction au sujet des objets d'art, mais comme en témoignent également d'autres concepts juridiques portant sur le collectif tels que les droits d'usage public ${ }^{28}$ et les intérêts diffus dans la tradition italienne et dans d'autres pays en Europe ${ }^{29}$ et en Amérique latine, ce caractère indéterminé du sujet collectif destinataire peut se révéler crucial pour mettre en évidence une destination plutôt qu'une titularité, une affectation et une finalité, plutôt qu'une seigneurie sur la chose qu'impliqueraient un sujet et un objet juridiquement déterminés. Dans la tradition italienne, les biens culturels attestent ainsi d'une vocation potentiellement dualiste de la propriété en vertu de cette mise en avant des finalités, indépendamment de l'introduction de la notion de patrimoine de la nation par la Constitution et donc de cet outil conceptuel, dont l'utilisation juridique ne se révèle jamais anodine (Cornu 2005). L'analyse ante litteram fonctionnaliste du droit de propriété forgée par la doctrine à partir des objets d'art constitue alors une première intuition interprétative, reprise ensuite et confirmée par la Constitution de 1948.

La Constitution italienne a d'ailleurs intégré d'autres régimes propriétaires particuliers comme en premier lieu, la propriété agricole inscrite (par l'art.44) dans un projet territorial de protection et de développement généraux et soumis en conséquence à de nombreuses limitations. C'est le dialogue entre la Charte constitutionnelle et les institutions propriétaires du monde rural que Salvatore Pugliatti élabore ainsi dans son modèle de pluralisme propriétaire. Dans son arbre des propriétés, devenu ensuite une référence pour d'éminentes doctrines civilistes, nous retrouvons plusieurs branches (unifiées par un tronc conceptuel commun) représentées par la multiplicité des institutions propriétaires. Dans ce contexte et parmi les biens fonciers collectifs, les propriétés collectives, la propriété agricole, et plus tard certaines formes de logement social, les biens culturels et paysagers trouvent ainsi parfaitement leur place en tant que propriétés fonctionnalisées.

Dans ce modèle propriétaire déjà amorcé auparavant pour les objets d'art et plus largement orienté socialement par le programme constitutionnel italien, l'exclusivité ne constitue pas un élément incontournable car de nombreuses propriétés sont inclusives par leur jouissance ou par leur finalité. À l'inverse, ce qui semble faire tronc commun dans cette interprétation pluraliste est d'une part le pouvoir de contrôle sur l'usage de la chose (Pugliatti 1964) ${ }^{30}$ et d'autre part, la fonction sociale qui dans cette vision constitue une limite interne au droit de propriété et non pas une limitation externe dictées par les mécanismes d'interdiction administrative ou procédurale. 
Figure 3

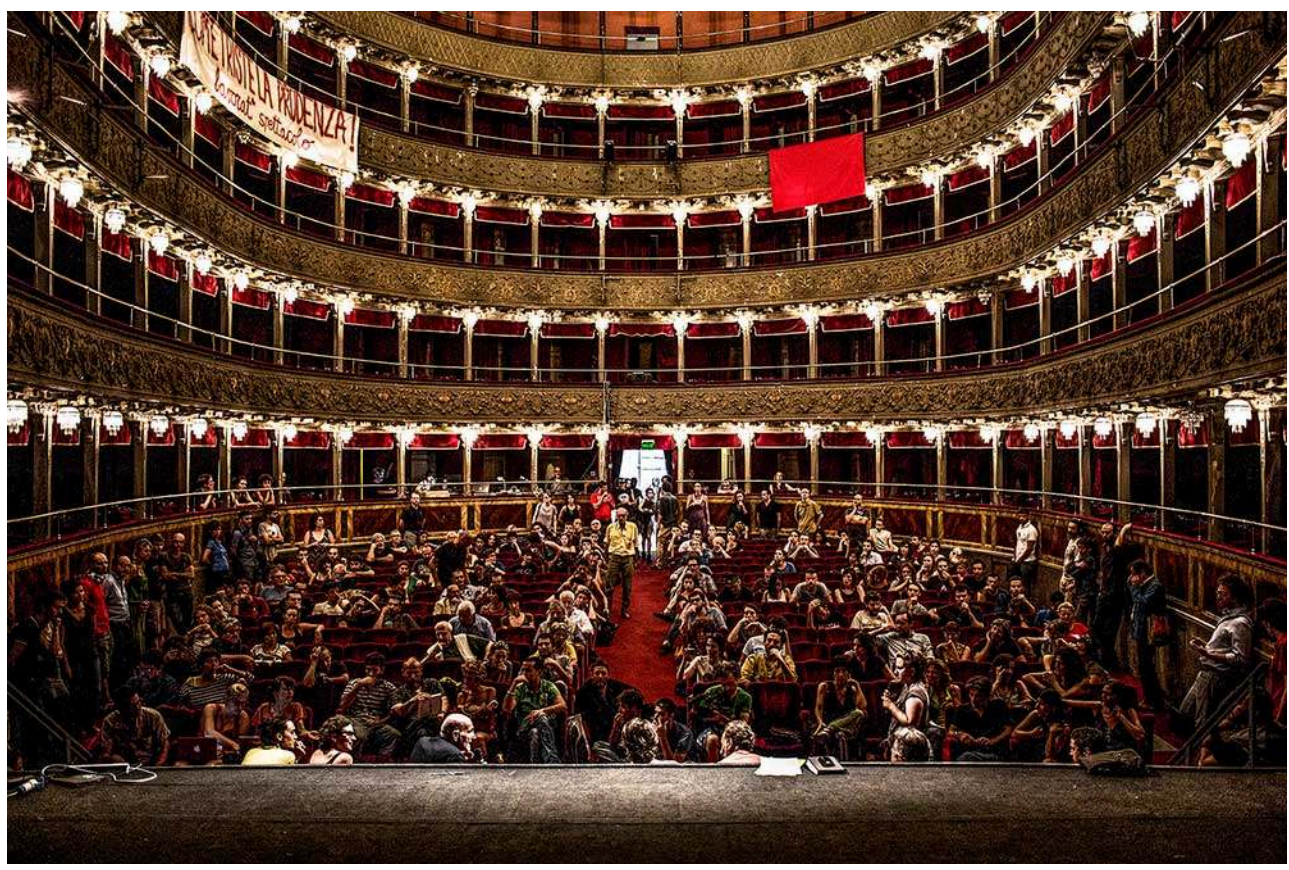

Pendant son occupation (2011-2014), le Teatro Valle devint un véritable laboratoire ouvert sur le thème des communs grâce à un calendrier dense d'assemblées publiques, de groupes de travail thématiques, de rencontres, de formations théoriques et pratiques articulés pour expérimenter des formes de gouvernance participatives. En 2014, le Teatro Valle Occupato reçu le prestigieux prix ECF Princess Margriet Award en raison de sa capacité à saisir « la culture comme un espace de possibilité. Un espace à travers lequel de nouvelles traditions de commoning 医 pratique de coopération sociale contre la privatisation croissante et la régulation descendante des ressources

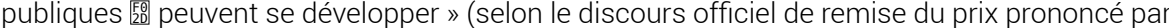
Katherine Watson, directrice de l'European Cultural Foundation, librement traduit par l'autrice).

(c) Valeria Tomasulo. 
Figure 4

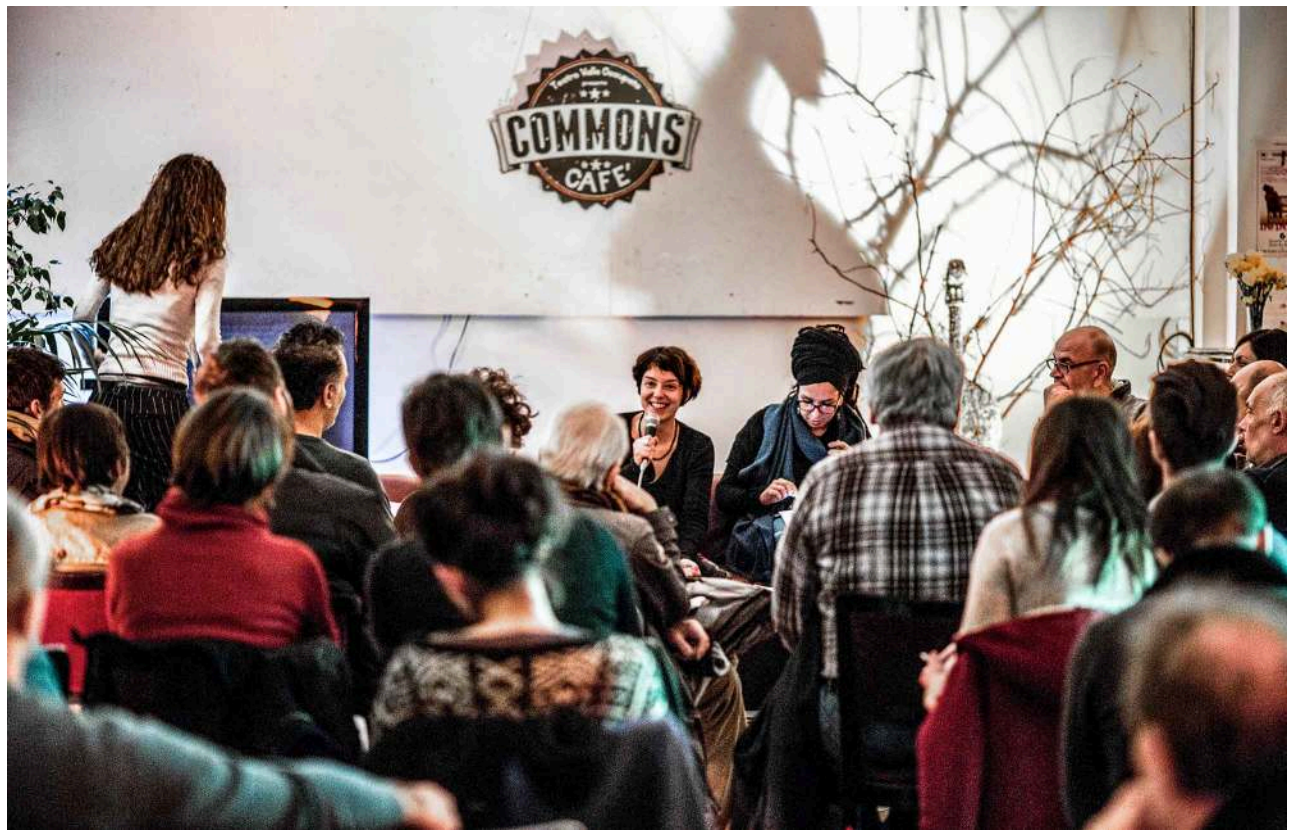

De nombreux « commons cafés » furent organisés au Teatro Valle pour mettre en relation les acteurs impliqués dans les différentes expériences de commoning en Italie et en Europe, échanger leurs expériences, se former réciproquement, créer des circuits de mutualisation et de collaboration, et discuter des propositions politiques et légales pour défendre et diffuser les commons.

(c) Valeria Tomasulo.

Figure 5

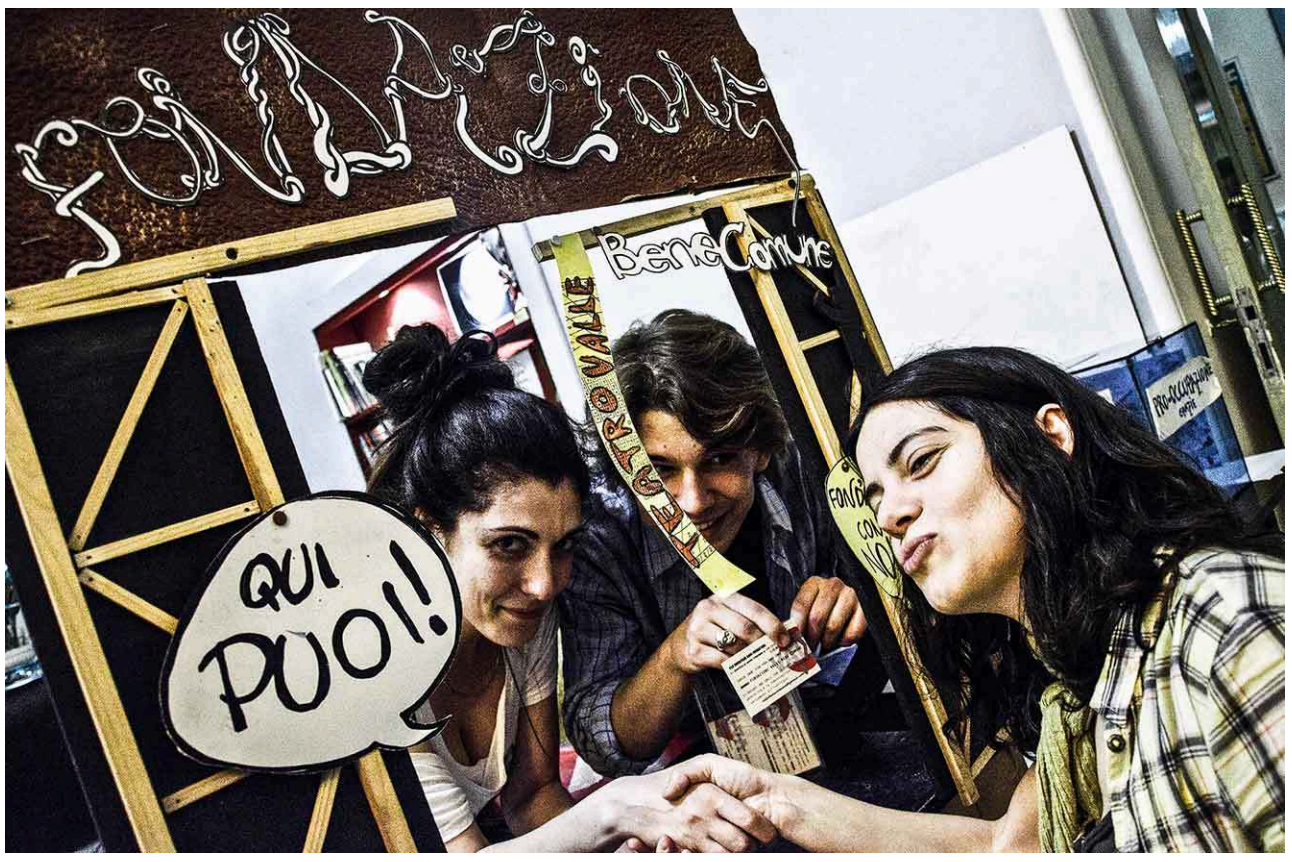

Une image de la campagne publique pour la création de la Fondation Teatro Valle Bene Comune qui, à travers un processus bottom-up, conduit en 2013 à la constitution participé d'un fond patrimonial et à l'élaboration d'un modèle atypique et original de fondation culturelle.

(c) Valeria Tomasulo. 


\section{La valorisation et l'« assaut »}

En Italie, les transformations qui ont affecté les visions et politiques du patrimoine dans les dernières décennies peuvent être lues à travers trois dynamiques souvent interdépendantes et parfois opposées: dénationalisation, valorisation, mise en commun.

\section{Dénationalisation et valorisation}

En arrière-plan de ce processus se déroule la parabole ascendante du néolibéralisme. Selon l'efficace analyse de Wendy Brown, la phase qui caractérise les économies capitalistes actuelles ne se manifeste plus uniquement par un recul de l'État vis-à-vis des acteurs économiques ou par la seule déresponsabilisation des institutions publiques par rapport à leur rôle dans un cadre de welfare state, mais plutôt par une extension des valeurs du marché aux politiques sociales et par l'introduction de segments importants de droits sociaux et de citoyenneté sur le marché (Brown 2003).

C'est dans la perspective plus large de cette extension de la logique économique et d'une rhétorique globale sur la valorisation du patrimoine qu'il nous faut ensuite lire les réformes advenues en Italie à partir des années 1990 et plus nettement au tournant des années 2000. La compétitivité touchera de façon emblématique les biens culturels, mais plutôt qu'une forme immédiate de privatisation, on observe un détournement dans une temporalité longue et comme une sorte de dé-publication ${ }^{31}$ ou, selon la définition de Saskia Sassen (2003), une dénationalisation progressive. Cette transformation des structures du pouvoir ne vise pas d'emblée la privatisation mais se caractérise d'abord par un transfert de certaines compétences auparavant strictement attribuées à l'État-nation vers des institutions à échelle urbaine ou internationale. La fabrique du patrimoine culturel ne se situe que partiellement au niveau national. Vue sous un angle conceptuel ou politique, cette notion est en fait un exemple typique de terrain hybride. Elle est à présent largement inscrite dans le cadre international et dans les politiques des collectivités territoriales responsables désormais de sa valorisation (conjointement avec les pouvoirs centraux) selon la répartition de compétences introduites par la réforme constitutionnelle italienne de 2000 qui réserve à l'État, de manière exclusive, les seuls pouvoirs de protection.

Dans les années 1990, dans l'objectif d'une valorisation globalement promue par les sources internationales, les réformes du secteur patrimonial (décret Ronchey, 1993 et décret Paolucci, 1998) avaient ouvert la voie au secteur privé dans la gestion de services concernant les biens culturels (par exemple dans les services muséaux et le tourisme culturel) afin de donner plus d'efficacité à un secteur alourdi par l'inertie des administrations publiques. À la suite de cette première phase, on enregistre en Italie un rapide passage à une valorisation d'ordre financière du patrimoine immobilier public largement constitué par des biens culturels et environnementaux - afin de répondre aux impératifs budgétaires de plus en plus sévères et aggravés par le fort endettement public.

Dans les années 2000, en concordance avec des orientations européennes et internationales nettement néolibérales, cette dynamique subira une accélération par des politiques dites de "finance créative» du gouvernement Berlusconi, mais 
prolongée bien au-delà de ce dernier. Ce qui a été pertinemment nommé «assaut contre le patrimoine ${ }^{32} »$ concerne cette deuxième tendance.

Si en 2009 le décret Ronchi s'attaque aux services publics et à l'eau en décrétant l'entrée massive des personnes privées dans ces secteurs, une réforme avait déjà institué en 2002 deux sociétés anonymes à contrôle public aux noms évocateurs: Patrimonio dello Stato SpA (dissous en 2011) et Infrastrutture SpA (acquise en 2006 par un fond public d'épargne). Leur but était de trouver les ressources nécessaires à la réalisation d'un ambitieux programme de grandes infrastructures par des techniques de valorisation inspirées du secteur privé, qui vont de la vente de nombreux biens immobiliers au seul transfert de la gestion des biens publics indisponibles. Cette réforme fut auparavant soigneusement préparée (D. 1.25 septembre 2001, n. 351) avec une vaste opération d'inventaire du patrimoine immobilier public qui dans les faits accordait aux fonctionnaires administratifs le pouvoir de décréter (ou implicitement de retirer) le caractère domanial ou indisponible des biens recensés. Comme l'a déjà souligné Paolo Grossi au sujet des réformes fascistes en matière de biens fonciers collectifs $^{33}$, ces montages masquent souvent des objectifs de liquidation. Ainsi, afin de «titriser» ("cartolarizzare ») pour ensuite privatiser les actifs immobiliers, le même décret envisageait la constitution de ces sociétés à responsabilité limitée ayant comme objet exclusif le placement de ces titres. Leurs activités, notamment celle de Patrimonio SpA concernera massivement le patrimoine de l'État et des collectivités territoriales et touchera d'innombrables biens culturels qui figuraient désormais, aux mêmes titres que d'autres actifs, parmi le patrimoine en recherche d'acquéreurs sur le site officiel du gouvernement.

\section{Mise en commun}

L'inquiétude généralisée suscitée par la mise en vente des actifs publics avait déjà conduit à l'institution par le Gouvernement Prodi de la Commission ministérielle sur les biens publics ${ }^{34}$ dont les conclusions innovantes avaient été saluées avec un large consensus dans le monde politique et académique. Toutefois, la non-discussion du texte proposé en 2008 par la Commission Rodotà et l'approbation par le nouveau gouvernement de mesures promouvant une privatisation généralisée, comme le décret Ronchi en 2009, ont provoqué de fortes mobilisations sociales.

C'est donc la combinaison des réactions aux privatisations des services publics (dont celui hydrique) et à la longue vague de déclassement, vente ou transfert massif de la gestion des biens du patrimoine culturel qui déclenchera - pendant les années de la crise et de l'austérité - un mouvement unitaire en Italie. Au sein d'un groupe hétérogène d'acteurs mobilisés convergeront des luttes d'ordre territorial préoccupées par leur milieu de vie, ainsi que des revendications des nouveaux secteurs professionnels émergents. Les associations culturelles, les comités locaux, les travailleurs précaires de la connaissance, de l'art et de l'information dénonceront alors une précarité à la fois sociale et territoriale, un manque d'accès tant aux lieux qu'aux services publics, désormais capturés par le processus de marchandisation généralisée ${ }^{35}$. Les emblèmes de cette unité seront le referendum contre la privatisation de "l'eaubien commun ${ }^{36}$ ", qui connaîtra une participation inédite et une mobilisation sociale et politique qui engendrera dans toute la Péninsule une vague d'occupation d'espaces publics abandonnés ou en cours de privatisation ${ }^{37}$. 
La notion de patrimoine culturel et plus généralement de patrimoine territorial comme bien commun constituera donc, au moins dans un premier temps, une réponse défensive à la privatisation des biens culturels et du patrimoine immobilier public. Le rapport qui accompagne le texte de la Commission Rodotà rend compte explicitement de cette préoccupation. De même, la décision de la Cour de cassation en 2011 évoque ponctuellement les étapes de ce processus de privatisation pour montrer l'insuffisance des catégories du code civil afin de cadrer les statuts et les régimes des biens à l'heure de cette profonde transformation dans les politiques qui les concernent. En réponse à cette urgence, certains biens sont désignés en tant que communs pour l'importance des finalités qu'ils engendrent et sont donc protégés du marché au-delà de leur titularité désormais incapable - bien que publique - d'en garantir la protection. Parallèlement à une liste non exhaustive de biens communs ${ }^{38}$ qui comprend parmi d'autres «les biens culturels, archéologiques et environnementaux et les zones paysagères protégées ", une formule ouverte définit ces biens comme "des choses qui expriment des utilités fonctionnelles à l'exercice des droits fondamentaux ainsi qu'au libre développement de la personne ». Ces droits fondamentaux seraient identifiés àtravers les principes fondamentaux de la Charte constitutionnelle qui place la personne humaine au cœur de son programme et, via la Constitution, également en relation avec les autres sources internationales portant sur les droits fondamentaux.

Cette référence à la personne est inscrite dans une perspective diachronique. Ces biens sont communs car ils développent leurs fonctions fondamentales dans le temps aussi bien pour les générations présentes que "pour les générations futures». Cette indication inspirée par l'élaboration sur les biens culturels et paysagers se reflète par conséquent sur l'ensemble des biens communs qui assument globalement cette fonction de transmission matérielle et symbolique traditionnellement réservée au patrimoine. L'accès à ce bien semble alors devenir la clef pour interpréter l'épanouissement de la personne comme un projet indissociablement matériel et immatériel dans une vision de l'égalité en tant que possibilité concrète et substantielle, tantôt pour la communauté présente, tantôt pour les générations futures ${ }^{39}$. Droits fondamentaux, protection des ressources territoriales et responsabilité intergénérationnelle deviennent alors les paramètres pour cadrer la notion d'accès aux biens communs. Un accès direct aux utilités des biens et non pas à la propriété, devient la voie pour garantir - ou au moins ne pas compromettre - le développement de la personne humaine.

41 Ainsi, dans l'approche italienne des biens communs, c'est en raison de cette fonction fondamentale que ces choses sont considérées communes et placées dans un espace protégé du marché et non pas en raison de leur gouvernance collective selon l'approche néo-institutionnaliste des communs de l'école de Bloomington ${ }^{40}$.

\section{Les expériences de commoning de biens culturels urbains}

42 Si l'intention primaire de la proposition Rodotà fut alors la sauvegarde de la destination sociale de certains biens, des voies ultérieures se dessinent dans les lignes du texte. "L'usage collectif doit être garanti dans les limites et selon les modalités fixées par la loi » affirme la proposition de projet de loi Rodotà qui aurait dû se traduire ensuite dans des décrets législatifs plus détaillés. L'usage collectif est donc posé comme finalité 
prééminente de ces biens qui ne doivent pas être simplement préservés en vue de leur transmission mais être effectivement accessibles. De plus, le rapport qui accompagne le texte envisage une connexion intime entre biens communs et participation directe. L'allusion renvoie d'abord aux articles constitutionnels consacrés à la démocratie directe (loi et referendum d'initiative populaire) mais le texte envisage également une large tutelle des biens communs en reconnaissant une légitimation populaire pour agir en justice et réclamer l'interdiction de tous comportements qui leur portent préjudice. Dans de nombreux écrits, les juristes de la Commission ${ }^{41}$ soulignent d'autres connexions étroites entre l'usage collectif et ces biens communs qui, en raison de leurs fonctions et grâce aux limitations conséquentes des pouvoirs du propriétaire, peuvent être mis dans une disponibilité collective effective. Néanmoins, l'extension de cette destination restait dans un premier temps encore à clarifier.

C'est au cours de cette mobilisation inédite désignée par Franco Cassano (2004) comme « la raisonnable folie des biens communs » que le lien entre cette notion et celles des communs se précisera grâce à une dialectique constante avec les acteurs sociaux engagés dans le commoning et la théorie des communs qui a désormais démontré la légitimité - juridique et économique - des formes de gouvernement collectif pour certaines ressources matérielles et immatérielles.

Trois expériences parmi les pratiques de réappropriation des espaces urbains qui se développent au sein du mouvement des communs en Italie, touchent plus particulièrement des objets patrimoniaux remarquables. Le théâtre Valle occupé à Rome, l'ex-Asilo Filangieri à Naples et la Cavallerizza Irreale de Turin revendiquent la gestion collective de bâtiments classés comme biens culturels et sont impliqués dans un dialogue direct avec les théoriciens du commun.

Revendiquant l'accès à des espaces urbains au nom de la "culture, bien commun ", l'un des plus importants théâtres "à l'italienne " du début du XVIII siècle est occupé au lendemain de la victoire du referendum pour l'«eau, bien commun» par les travailleuses et travailleurs de l'art et de la culture. Ils lancent avec succès la campagne "Teatro Valle bene comune" pour constituer une "fondation de participation" (fondation atypique) pour une gestion de type collectif, ouverte à la participation directe des citoyens ${ }^{42}$. La forme juridique, pour enraciner ce commun urbain, est ici davantage recherchée dans l'espace d'autonomie collective garantie par le droit privé.

À Naples, l'une des expériences les plus anciennes du mouvement porte sur l'ex-Asilo Filangieri, un ancien couvent classé appartenant à la Ville et jusqu'alors largement sous-utilisé. L'expérience qui rencontre un grand consensus trouve également l'intérêt de l'administration municipale qui avait articulé sa propre campagne électorale sur le thème des biens communs en soutenant des politiques et un assessorat ad hoc. La stratégie juridique envisagée pour régulariser l'expérience porte sur une reprise des «droits civiques d'usage» suivant une relecture de l'art. 43 de la Constitution et se situe donc plutôt sur le versant du droit public. En 2012 et à la suite de l'occupation de l'immeuble, la Ville de Naples reconnaît effectivement l'ancien asile comme un bien commun pour ses fonctions et admet une gestion participative de la communauté ouverte à l'origine de la réappropriation de l'édifice. Dans les délibérations municipales, cette collectivité est désignée comme "communauté des travailleurs de l'immatériel » en cohérence avec le profil des acteurs engagés dans l'expérience. Ce transfert à une collectivité informelle se fonde également sur une interprétation renouvelée de l'art. 43 de la Constitution. L'usage de l'immeuble lui est concédé à titre 
gratuit et sans aucune redevance ; la prise en charge des frais d'utilisation, de conciergerie et de gardiennage étant garantie par la municipalité. La communauté s'engage à formuler un règlement établissant des règles d'accès, de partage et de décision en s'inspirant des droits d'usage collectifs traditionnellement autoréglementés par la communauté. Après trois ans d'expérimentation et d'écriture collective, la Déclaration de droit civique et collectif d'usage proposée sera validée dans une délibération ( $\left.n^{\circ} 893 / 2015\right)$ de la Ville.

\section{Figure 6}

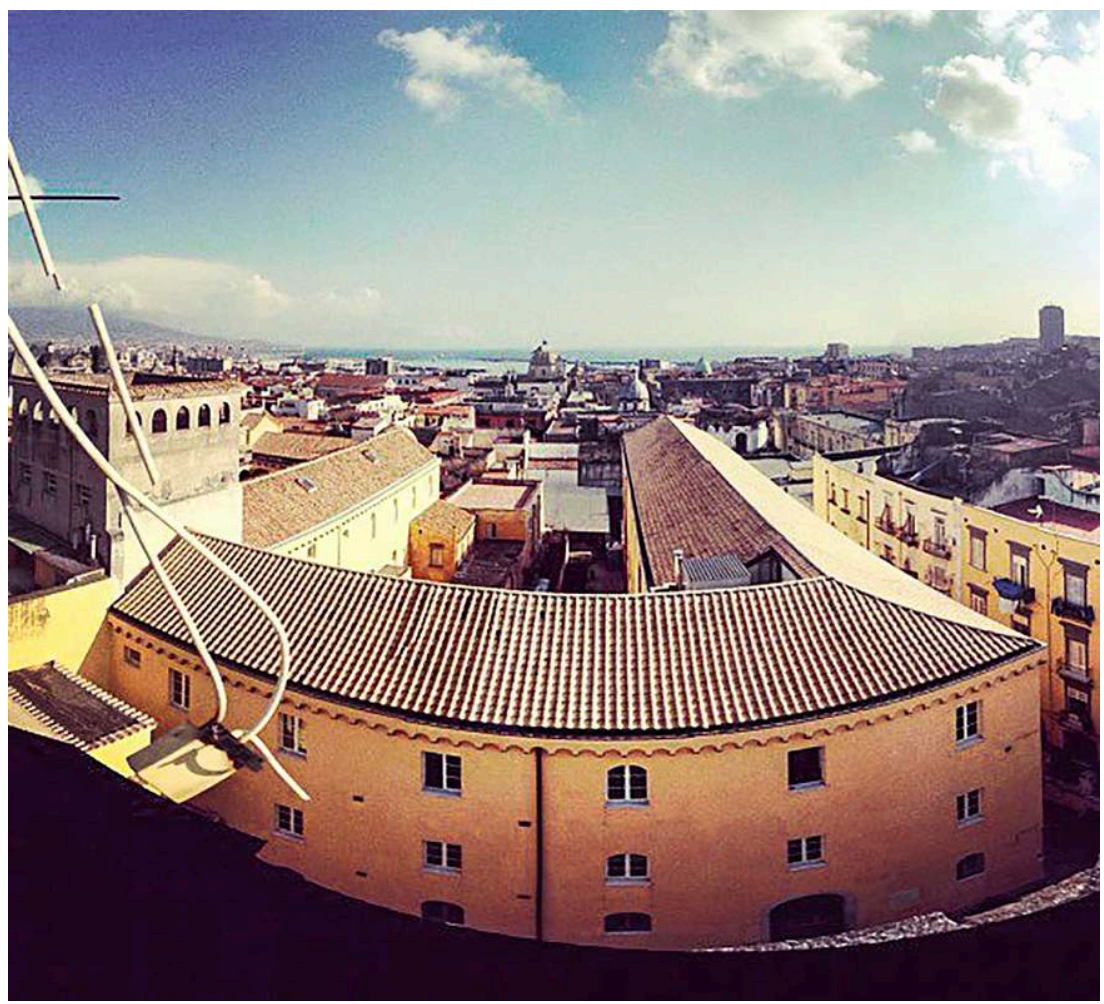

L'ex-Asilo Filangieri, situé dans le cœur de la ville historique de Naples, était à l'origine un monastère créé durant la première moitié du xvi siècle par des religieuses d'Europe de l'Est. Ses fondations reposent sur un site archéologique dont de nombreux éléments semblent remonter au ıve siècle. Après la Première Guerre mondiale, le bâtiment fut acheté par la comtesse Giulia Filangieri di Candida qui le transforma en orphelinat. L'asile resta actif jusqu'en 1980, année du séisme qui toucha gravement la région. En état d'abandon, il devient le théâtre de trafics et de criminalité jusqu'à sa restauration en 2005. En 2009, la municipalité attribua l'ensemble à la Fondazione Forum delle Culture 2013, un organisme soupçonné d'être un lieu de drainage de fonds publics et de gestion clientéliste du consensus qui en fit usage de manière discontinue, sans projet culturel clair.

(c) L'Asilo, ex-Asilo Filangieri. 
Figure 7

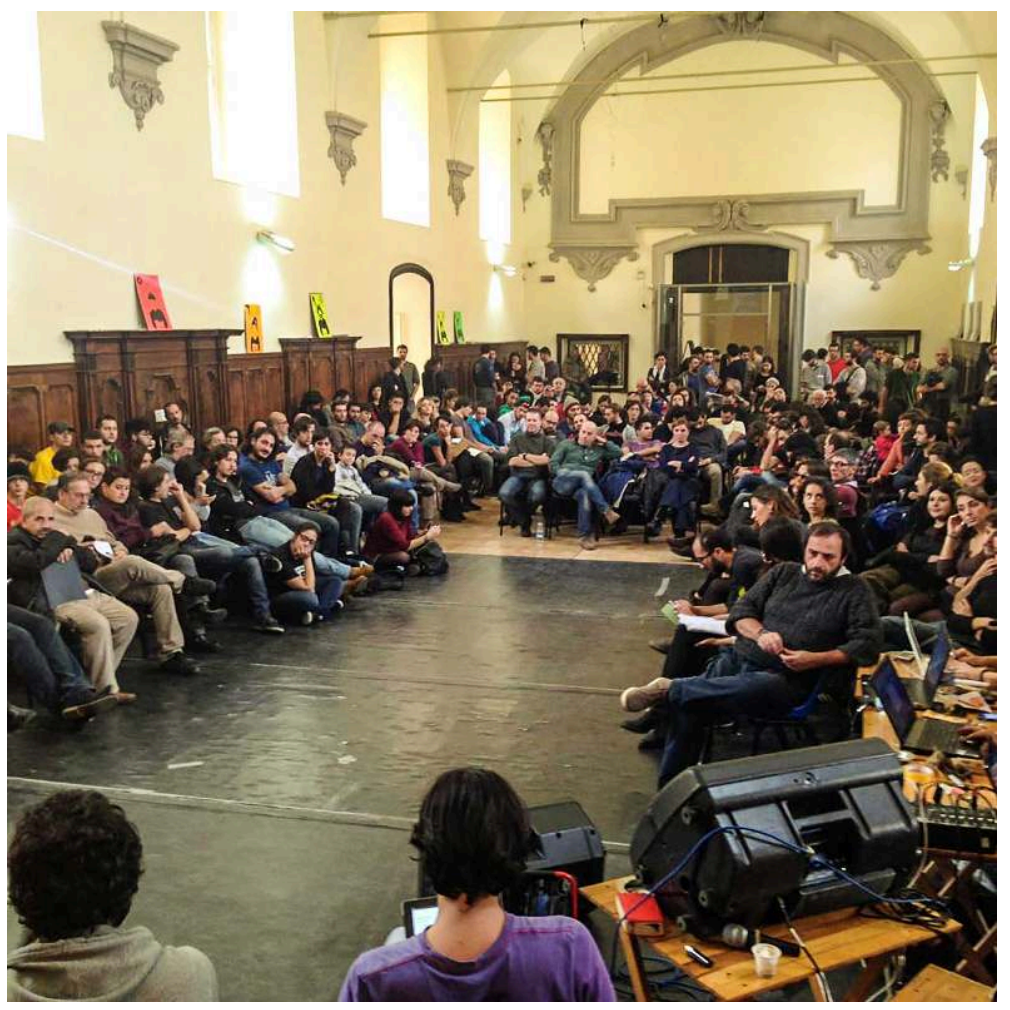

En 2012, l'ex-Asilo Filangieri, fut occupé par La Balena, collectif de travailleuses et de travailleurs de l'art et de la culture, qui revendiquait un accès et un usage collectif de ce lieu supposément consacré à la culture mais, dans les faits, gravement sous-utilisé et inaccessible aux citoyens. Le groupe s'élargit ensuite considérablement et lança un vaste projet de participation citoyenne ainsi qu'une interlocution avec la mairie nouvellement élue, autour d'un programme de protection et d'accessibilité des biens communs. Ici, l'une des nombreuses assemblées organisées au cours du processus participatif développé pour décider les formes de gouvernance de l'Asilo et en rédiger le règlement d'usage, ainsi que pour définir le rôle des différents groupes d'usagers impliqués.

(c) L'Asilo, ex-Asilo Filangieri. 
Figure 8

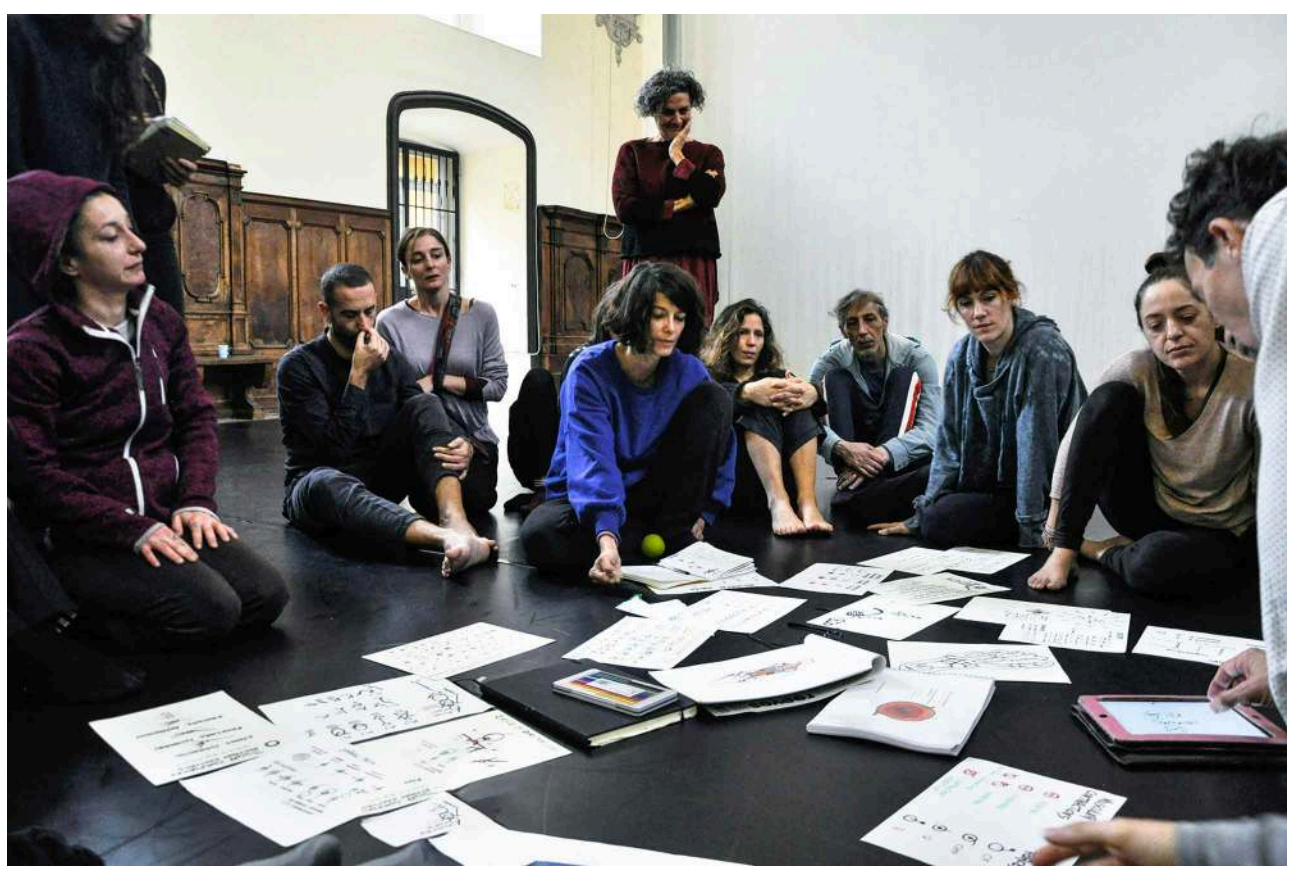

Atelier du danseur, pédagogue et chorégraphe de renommée internationale, Frey Faust, dans les espaces de l'ancien réfectoire de l'ex-Asilo Filangieri. La salle est équipée des bancs en bois d'origine et réaménagée 医 grâce à des interventions modulaires et souples crées in situ par les professionnelles des métiers de la scène théâtrale de l'Asilo [0: travail scénique un confort maximal sans dégrader la qualité patrimoniale des lieux, et en intégrant ses éléments dans le paysage imaginaire de la production artistique à la une du programme. L'Asilo propose de nombreux ateliers de formation professionnelle dirigés par d'importants artistes et créateurs internationaux, avec une politique d'accessibilité des tarifs, de transversalité entre disciplines et savoirs, et d'ouverture aux citoyens.

(c) Ivana Fabbricino. 


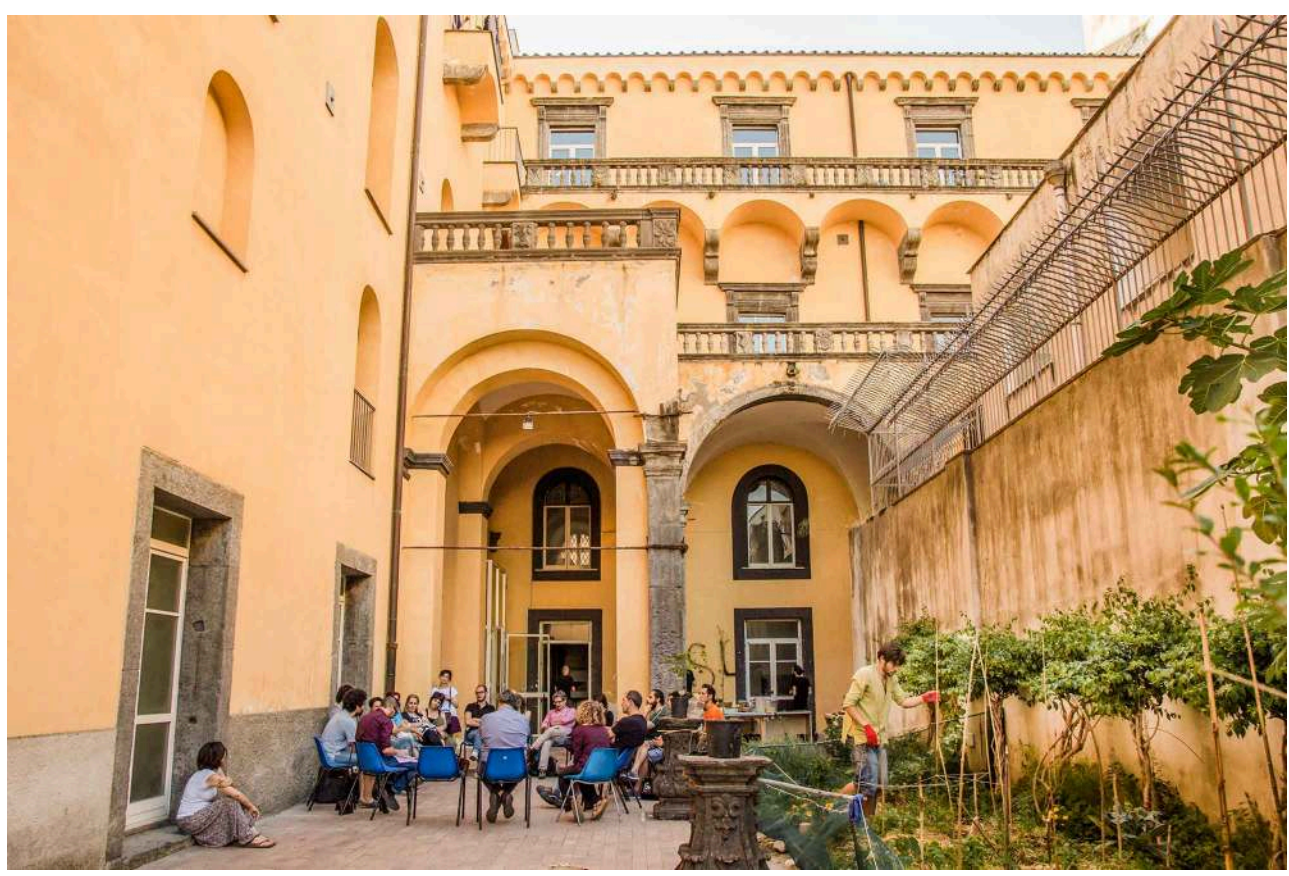

Dans la cour de l'ex-Asilo Filangieri, deux exemples d'usages différents et simultanés de l'espace commun : parallèlement à la tenue d'une réunion du groupe responsable de la programmation culturelle de l'Asilo, prennent place les activités de culture botanique dans l'aire destinée à devenir un jardin partagé et pédagogique.

(c) Sabrina Merolla.

De lege lata, aucun droit d'usage ne peut être en réalité revendiqué dans le lieu en question sur la base de la loi fasciste de 1927 qui règle ces usages et les configure comme des droits réels collectifs ancrés dans le passé. La référence à ces usages réadaptés au milieu urbain marque plutôt la volonté de réhabiliter la notion d'usages collectifs, qui occupent comme on l'a vu une place importante dans notre tradition. Le schéma de propriété divise, là où la titularité peut se réduire à une simple propriétéfonction ${ }^{43}$ et légitimer, par exemple, l'accès aux demeures historiques en milieu urbain (telle que la Villa Borghèse), permet effectivement d'envisager un accès et un usage collectif sur un bien destiné. Cette destination reposerait alors sur l'identification de l'ex-Asilo Filangieri comme bien commun, identification effectuée par la Municipalité sur sollicitation d'une communauté de citoyens. De plus, la légitimation officielle par la Ville accompagnée par une grande vitalité de l'expérience, intronise l'ex-Asilo comme modèle d'urban commons auquel beaucoup d'acteurs sociaux comme de villes italiennes et européennes s'intéressent de près.

Les impressionnantes écuries de la Cavallerizza de Turin sont occupées quant à elles dès 2014 lors de leur transfert du patrimoine de la Ville à la société anonyme locale afin de les titriser, puis de les vendre comme actifs municipaux. En parallèle, les délibérations nécessaires pour déclasser ces édifices étaient en cours d'approbation par le conseil municipal. Depuis, la Cavallerizza Irreale s'oriente dans la même direction que l'ex-Asilo de Naples mais sans avoir encore reçu une légitimation similaire par la Ville. Elle adopte un modèle d'organisation semblable et une argumentation juridique analogue pour proposer l'usage civique collectif du complexe immobilier faisant partie des anciennes résidences de Savoie déclarées patrimoine Unesco en 1997. 

règlements municipaux relatifs aux biens communs urbains diffusés en Italie dans la même période ${ }^{44}$ s'intéressent à des questions patrimoniales. Dans la plupart de ces chartes, la place occupée par les citoyens est strictement réglée sous le prisme de la concession administrative et se détache de la recherche juridique plus articulée sur les biens communs pour développer des outils de gouvernance locale. Néanmoins, il faut signaler que le mécanisme d'individuation des biens matériels ou immatériels qualifiés de communs urbains, en vertu d'une initiative municipale ou sur proposition citoyenne, n'exclut pas qu'ils puissent être classés. De manière plus spécifique, les règlements d'Orvieto et de Pérouse font référence à la convention de $\mathrm{Faro}^{45}$ comme une des sources favorisant un investissement direct des habitants dans la protection du patrimoine voire une participation à la gestion de certains lieux par des groupes mêmes informels en tant que "communautés patrimoniales" (selon l'expression de la convention). De plus, la charte de Pérouse envisage des pouvoirs plus conséquents des citoyens (demande d'information et de motivation publique de tous actes ou omissions) envers la mairie en raison de ses compétences en matière de classement ${ }^{46}$.

Dans le débat désormais global traitant des communs urbains ${ }^{47}$, des expériences de commoning touchant à des biens culturels sont d'ailleurs aussi présentes dans d'autres pays comme dans la ville belge de Gand qui affiche une politique spécifique autour des communs. Parmi d'autres expériences promues par la Municipalité, l'ancienne abbaye Saint-Bavon, site le plus ancien de la ville, auparavant abandonné, est géré depuis 2007 par un groupe de citoyens. Constitué ensuite en organisation sans but lucratif, le collectif des voisins de l'abbaye assure l'ouverture au public et de nombreuses activités culturelles in situ ${ }^{48}$. Dans cette expérience, le schéma de l'attribution en gestion à la communauté locale a évolué d'une attribution à un groupe informel vers un modèle plus classique ${ }^{49}$ : la Ville délègue le sujet juridique collectif gérant et intermédiaire pour permettre l'accès de la plus large communauté urbaine qui reste sujet-tiers bénéficiaire.

51 Le lien entre la notion de biens culturels / biens communs en tant que biens fonctionnels pour la préservation et l'accès au patrimoine culturel et les communs comme forme collective de gestion se fait donc en premier lieu par le dépassement de la dichotomie public-privé et l'ouverture d'une troisième voie. Les études de Elinor Ostrom, qui ont connu une grande diffusion dans ces mêmes années ${ }^{50}$, ont en fait prouvé la possibilité que le gouvernement collectif de certaines ressources puisse être parfaitement efficace et durable, contredisant ainsi les «tragiques" prédictions de Garrett Hardin.

Dans une perspective plus radicale, les expériences signalées et souvent animées par des précaires de la recherche, de l'art et de la culture, développent une idée émancipatrice de ces lieux en les considérant non seulement comme patrimoine culturel à rendre accessible mais aussi comme fabrique de l'immatériel. Rendus disponibles aux acteurs du secteur cognitif, les arts et les connaissances trouveraient dans ces lieux non seulement des espaces de représentation, mais également de reproduction. On parlera de «biens du patrimoine de l'immatériel » en mettant en évidence la connexion entre lieux culturels et production contemporaine selon un axe déjà parfaitement saisi par les politiques de marketing territorial ${ }^{51}$.

53 Ces lignes de fuite de la dichotomie public-privé demeurent ainsi dans cet espace ambivalent habité par les villes dans leurs nouveaux modèles de gouvernances globales. 
Le risque majeur est que ces communs urbains s'inscrivent dans une tendance au recul et à la déresponsabilisation des institutions territoriales, et que l'activisme social soit l'instrument $\mathrm{du}$ démantèlement $\mathrm{du}$ welfare local à défaut d'un développement personnel et social. Dans le cas italien par exemple, le transfert d'une partie des compétences concernant la valorisation patrimoniale de l'État aux villes a représenté une schizophrénie du système faisant proliférer des approches incohérentes et contradictoires des mêmes objets patrimoniaux. D'une part, les collectivités territoriales se sont vues attribuer ces pouvoirs sans développer de réelles compétences spécifiques et ont souvent géré ces biens avec les mêmes logiques adoptées dans les autres secteurs urbains (en leur appliquant par exemple les mêmes mécanismes d'appels d'offre élaborés pour les services). D'autre part, en relativisant l'État ${ }^{52}$ et le caractère plus figé de ses procédures, les compétences décentrées, la diffusion de formes de gouvernance urbaine et de soft law local ont indirectement ouvert le champ des possibilités. De ces ambiguïtés ont émergé des expérimentations locales innovantes, comme celles de Naples, montrant que l'accès aux biens culturels pouvait se coordonner avec des modes de gestion collective sans compromettre leur protection d'une part, et en favorisant largement leur accessibilité d'autre part, grâce à leur inscription dans le tissu vivant de la production culturelle contemporaine.

Figure 10

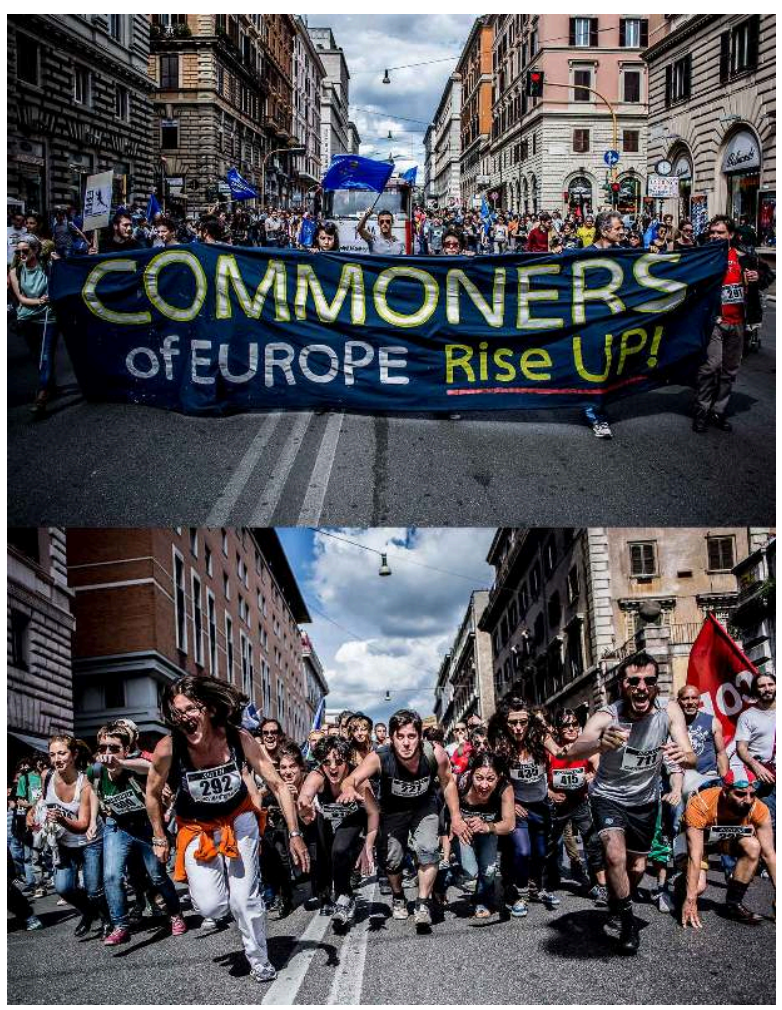

Rome, 17 mai 2014 : une des manifestations nationales organisées pour réclamer et défendre les communs qui ont ponctuées le Mouvement des communs en Italie, à partir de 2011, et dans lequel les travailleuses et travailleurs du spectacle et de la culture, réunis autour du Teatro Valle et autres lieux culturels occupations en Italie, ont joué un rôle clé.

(c) Photos Valeria Tomasulo. 


\section{Conclusions}

\section{introduction. Concernant la qualification des biens culturels comme biens communs} dans la tradition juridique italienne, nous pouvons sans hésitation confirmer cette hypothèse. L'éminente doctrine Rodotà mentionne directement les biens culturels et paysagers dans la liste des biens communs au-delà même de toutes interprétations ultérieures. De plus, la décision des Sections réunies de la Cour de cassation de 2011, bien que concernant un bien paysager, peut fournir une indication claire également en matière de biens culturels en raison de sa vocation générale et en raison de leur stricte connexion avec les biens paysagers, mentionnée par la Cour. La qualification des biens communs impliquerait d'après le texte de la Commission Rodotà que leur usage collectif soit garanti et que les biens soient gérés par des entités publiques et situées hors du commerce, si les titulaires sont des personnes morales de droit public, la concession devrait être admise dans les seuls cas prévus par la loi et pour une durée limitée, sans possibilité de prorogation. Une gestion inspirée par les principes de participation est préconisée pour assurer que leur destination et leur accès collectifs puissent rester effectifs dans le temps.

Un « bien culturel », une fois désigné comme bien commun, doit s'orienter vers l'usage collectif, si l'intégration des principes de participation peut être souhaitée de manière générale et à différentes échelles, et une gestion collective et participative n'en est pas une conséquence directe et immédiate. Toutefois et dans ce moment de relativisation de la seule primauté étatique dans la réalisation des intérêts généraux et de remise en question du système de la représentation, le gouvernement commun de biens culturels semble désormais une possibilité solide pour garantir le contrôle démocratique continu $^{53}$ sur leur destination collective comme des initiatives citoyennes l'ont d'ailleurs montré dans plusieurs contextes. Si une forme de gouvernement des biens culturels intégrant la participation citoyenne est aujourd'hui devenue un objectif politique légitime, la théorie des communs et la multiplication des expérimentations urbaines montrent, dans les faits, que, en présence d'une communauté active et disponible, leur gestion véritablement commune peut ainsi être socialement générative tout en représentant une solution parfaitement cohérente avec leur préservation et leur accès diffus.

Quant aux relations entre biens culturels et inclusivité, nous devons d'abord préciser que l'inclusivité peut être considérée, dans un sens plus strictement juridique, comme un paradigme émergent ${ }^{54}$ alternatif à l'exclusivisme qui caractérise traditionnellement la propriété en tant que relation aux autres concernant une chose ${ }^{55}$. Selon la proposition de Séverine Dusollier (2015 : en part. 104-105; 2020) ${ }^{56}$, nous pouvons alors représenter les relations propriétaires sur un continuum composé par des solutions qui combinent exclusivité et inclusivité de manière variable selon les objets sous-jacents.

Dans le schéma interprétatif de la propriété comme bundle of rights proposé par Hohfeld et remobilisé par Dusollier \& Rochfeld (2017) en développant une intuition de Yockaï Benkler (2014 : 10-11, 69-99), les œuvres intellectuelles, intrinsèquement vouées à la diffusion, pourraient par exemple constituer des propriétés inclusives (Dusollier \& Rochfeld 2017) car l'exclusivité jouerait un rôle secondaire par rapport à la préservation, à la circulation et à la jouissance de ces objets. Lorsque l'utilisation (même partielle) de certaines ressources ne peut pas, ou n'a pas de raison, d'être

In Situ. Au regard des sciences sociales, 2 | 2021 
interdite à un groupe de personnes, elles revêtent, de fait, un caractère commun. En désactivant l'opposabilité erga omnes du titre de propriété, les prérogatives concernant ce type de communs, seraient alors à interpréter comme des privilèges déterminés, selon Hohfeld, par l'absence d'un pouvoir du propriétaire de faire recours à l'État pour opposer à quiconque son droit exclusif. Le corrélatif du privilège est, en fait, le no-right dans la grille conceptuelle de Hohfeld. Cette interprétation admet le paradigme de l'inclusivité, comme conséquence de la paralysie (no-right) de l'opposabilité des prérogatives propriétaires, par rapport à certains biens, vis-à-vis des tiers et, parfois, vis-à-vis d'une communauté entière. Bien que se retrouvant de fait limité sur le plan opérationnel et quant à son impact social, le droit de propriété garde ainsi sa propre unité.

De manière analogue, l'exclusivité ne constitue pas un élément incontournable dans le modèle italien de l'arbre des propriétés, mais en raison d'arguments juridiques très différents: de nombreuses propriétés sont ici fonctionnalisées et, selon le modèle auparavant déjà évoqué pour les objets d'art, celles-ci peuvent être inclusives car leur jouissance est divisée entre le titulaire et la collectivité des usagers. Au niveau descriptif, l'élément commun à ces multiples propriétés reste le pouvoir de contrôle sur l'usage de la chose (qui n'exclut donc pas un usage partagé ${ }^{57}$ ) tandis que, selon une lecture systématique, une prééminente fonction sociale intervient, ainsi, comme limite interne au droit de propriété et de sa configuration substantielle et non pas comme une limitation externe opérante selon les mécanismes de l'interdiction administrative ou de la non-opposabilité.

La propriété sur des biens ayant une valeur culturelle et paysagère fondamentale est déjà fonctionnalisée et compressée lorsqu'elle se perfectionne ou se transmet au sein d'une telle architecture juridique. Le droit de propriété - public ou privé - sur les biens culturels est donc substantiellement inclusif car ces biens sont préordonnés au développement culturel de la communauté nationale et à la transmission aux générations futures et enfin, en tant que biens communs, destinés aux usages collectifs et aux « intérêts fondamentaux de tous les citoyens ${ }^{58}$ ».

\section{BIBLIOGRAPHIE}

AGIOLINI Chiara, 2017, « Intérêts diffus » [notice], in CORNU Marie, ORSI Fabienne \& ROCHFELD Judith (dir.), Dictionnaire des biens communs, Paris, PUF, coll. « Quadrige », p. 698-702.

ARAGÃO Alexandra \& CARVALHO Ana Celeste, 2017, « Taking access to justice seriously: diffuse interests and actio popularis. Why not? ", Environmental law network international review, $\mathrm{n}^{\circ} 2$, p. 42-48. Disponible en ligne, https://www.elni.org/fileadmin/elni/dokumente/Archiv/2017/ Heft_2/elni2017-2_Aragao_etal.pdf [lien valide en juin 2020].

BARTOLINI Antonio, 2013, « Beni culturali » [notice], in Enciclopedia del diritto. Annali, vol. 6, Abitazione. Volontariato, Milan, Giuffrè, p. 93-132. 
BENKLER Yochaï, 2014, « Between spanish huertas and the open road. A tale of two commons?», in FRISCHMANN Brett M., MADISON Michael J. \& STRANDBURG Katherine Jo (dir.), Governing knowledge commons, New York, Oxford University Press, p. 69-99.

BOCCON-GIBOD Thomas, 2014, « Duguit, et après ? Droit, propriété et rapports sociaux », Revue internationale de droit économique, t. 28, vol. 3, « Repenser la propriété », p. 285-300. Disponible en ligne, https://www.cairn.info/revue-internationale-de-droit-economique-2014-3-page-285.htm [lien valide en juin 2020].

BROWN Wendy, 2003, «Neoliberalism and the end of liberal democracy », Theory \& Event, vol. 7, $\mathrm{n}^{\circ} 1$ [en ligne], https://muse.jhu.edu/article/48659 [lien valide en juin 2020].

CALAMANDREI Piero, 1955, « La Costituzione », in COLLECTIF, Dieci anni dopo. 1945-1955. Saggi sulla vita democratica italiana, Bari, Editori Laterza, p. 209-316.

CALATA Pierre, 1966, « La transformation du patrimoine dans le droit civil moderne », Revue trimestrielle de droit civil, $\mathrm{n}^{\circ} 64$, p. 185 et sq.

CASSANO Franco, 2004, Homo civicus. La ragionevole follia dei beni comuni, Bari, Dedalo, coll. « Strumenti / scenari ».

CEDERNA Antonio, 2013, Lo Sfacelo del bel paese, Naples, La Scuola di Pitagora, coll. « Pan ».

CERULLI IRELLI Vincenzo, 1983, Proprietà pubblica e diritti collettivi, Padoue, Cedam, coll. « Pubblicazioni dell'Istituto di diritto pubblico della Facoltà di giurisprudenza dell'Università degli studi di Roma ».

CERULLI IRELLI Vincenzo, 1994, « I beni culturali nell'ordinamento italiano », Studi parlamentari e di politica costituzionale, $\mathrm{n}^{\circ} 103$, p. 27 et sq.

CHARMONT Joseph, 1903, « La socialisation du droit (leçon d'introduction d'un cours de droit civil) », Revue de métaphysique et de morale, t. 11, n 3, p. 380-405.

COHEN Morris R., 1927, « Property and sovereignty », Cornell Law quarterly, vol. 13, n 1, p. 8-30.

CORNU Marie, 2005, « À propos de l'adoption du Code du patrimoine. Quelques réflexions sur les notions partagées », Recueil Dalloz, n²2, p. 1452-1458.

CORNU Marie, 2014, « Du monument à l'œuvre d'art : le concept de propriété artistique à partir d'une lecture de Saleilles ", in BERNAULT Carine, CLAVIER Jean-Pierre, LUCAS-SCHLOETTER Agnès et al. (dir.), Mélanges en l'honneur du professeur André Lucas, LexisNexis, p. 185-196.

DARDOT Pierre \& LAVAL Christian, 2014, Commun. Essai sur la révolution au XXI siècle, Paris, La Découverte.

DI ROBILANT Anne, 2013, « Property: a bundle of sticks or a tree? », Vanderbilt Law review, vol. 66, $\mathrm{n}^{\circ} 3$, «Category », p. 869-932.

DUSOLLIER Séverine, 2015, «Inclusivity in intellectual property », in DINWOODIE Graeme B. (dir.), Intellectual property and general legal principles. Is IP a lex specialis?, Cheltenham, Edward Elgar Publishing, coll. « ATRIP intellectual property series », p. 101-118.

DUSOLLIER Séverine, 2013, « The commons as a reverse intellectual property. From exclusivity to inclusivity », in HOWE Helena R. \& GRIFFITHS Jonathan (dir.), Concepts of property in intellectual property law, Cambridge, Cambridge University Press, coll. « Cambridge intellectual property and information law », p. 258-281. 
DUSOLLIER Séverine \& ROCHFELD Judith, 2017, « Propriété inclusive ou inclusivité » [notice], in CORNU Marie, ORSI Fabienne \& ROCHFELD Judith (dir.), Dictionnaire des biens communs, Paris, PUF, coll. « Quadrige », p. 983-987.

DUSOLLIER Séverine, 2020, «Intellectual property and the bundle-of-rights metaphor », in DRAHOS Peter, GHIDINI Gustavo \& ULLRICH Hanns (dir.), Kritika. Essays in intellectual property, vol. 4, Cheltenham, Edward Elgar Publishing, p. 146-179.

FERRARI Mario, 2016, «Proprietà e diritto a essere inclusi », Rivista criticadel diritto privato, $\mathrm{n}^{\circ} 4$, p. 525-578.

FESTA Daniela, 2016, « Les communs urbains. L'invention du commun » [in dossier « L'Italie des biens communs » dirigé par CHARBONNIER Pierre \& FESTA Daniela], Tracés, n 16, numéro horssérie, « traduire et introduire », p. 233-256. Disponible en ligne [version longue], https:// journals.openedition.org/traces/6636 [lien valide en juin 2020].

FESTA Daniela, 2017a, « Biens communs en Italie, le mouvement social » [notice], in CORNU Marie, ORSI Fabienne \& ROCHFELD Judith (dir.), Dictionnaire des biens communs, Paris, PUF, coll. « Quadrige », p. 107-111.

FESTA Daniela, 2017b, « La Constituante des biens communs » [notice], in CORNU Marie, ORSI Fabienne \& ROCHFELD Judith (dir.), Dictionnaire des biens communs, Paris, PUF, coll. « Quadrige », p. 298-303.

FOSTER Sheila R., 2011, « Collective action and the urban commons », Notre-Dame Law review, vol. $87, \mathrm{n}^{\circ} 1$, p. 57-134.

FOSTER Sheila R. \& IAIONE Christian, 2016, « The city as a commons », Yale Law \& Policy review, vol. $34, n^{\circ} 2$, p. 281-349.

GAMBARO Antonio, 2012, « I beni » [notice], in SCHLESINGER Pietro, MESSINEO Francesco, CICU Antono \& MENGONI Luigi (dir.), Trattato di diritto civile e commerciale, Milan, Giuffré.

GAMBI Lucio, 1975, Una politica per I beni culturali. Stato e regioni. Incontro col ministro Giovanni Spadolini. Bologna, Archiginnasio 15 febbraio 1975, Bologne, Regione Emilia-Romagna Giunta, coll. «Quaderni della giunta régionale », p. 34-37.

GIANNINI Massimo Severo, 1976, «I beni culturali », Rivista trimestrale di diritto pubblico, vol. 26, p. 3-38.

GRISOLIA Mario, 1952, La Tutela delle cose d'arte, Rome, Società editrice del "Foro Italiano", coll. « Biblioteca del "Foro italiano" ».

GROSSI Paolo, 2017, « Droits fonciers collectifs » et « Droits civiques d'usage » [notices], in CORNU Marie, ORSI Fabienne \& ROCHFELD Judith (dir.), Dictionnaire des biens communs, Paris, PUF, coll. « Quadrige », p. 448-453 et p. 439-444.

HOHFELD Wesley Newcomb, 1913, « Some fundamental legal conceptions as applied in judicial reasoning ", Yale Law journal, vol. 23, p. 16-59. Disponible en ligne, https:// digitalcommons.law.yale.edu/cgi/viewcontent.cgi?article=2324\&context=ylj [lien valide en juin 2020].

HOHFELD Wesley Newcomb, 1917, « Fundamental legal conceptions as applied in judicial reasoning ", Yale Law journal, vol. 26, p. 710-770.

HUGO Victor, 1832, « Guerre aux démolisseurs », Revue des Deux Mondes. 
KENNEDY Duncan, 2006, « Three globalizations of law and legal thought: 1850-2000 », in TRUBECK David M. \& SANTOS Alvaro (dir.), The New Law and economic development. A critical appraisal, Massachusetts, Cambridge University Press, p. 19-73.

LUCARELLI Alberto, 2019, « Les nouvelles sources du droit public : participation et biens communs ", in RAPP Lucien, HAMDOUNI Saïd \& GUIGNARD Didier (dir.), Culture Société Territoires. Mélanges en l'honneur du professeur Serge Regourd, Bayonne, Institut universitaire Varenne, coll. «Colloques \& essais ».

MACPHERSON Crawford Brough, 1962, The Political Theory of possessive individualism. Hobbes to Locke, Oxford, Clarendon Press.

MAGNAGHI Alberto, 2014, La Biorégion urbaine. Petit traité sur le territoire bien commun, Paris, Eterotopia France, coll. « Rhizome ».

MARELLA Maria Rosaria, 2016, «La propriété reconstruite : conflits sociaux et catégories juridiques » [in dossier « L'Italie des biens communs » dirigé par CHARBONNIER Pierre \& FESTA Daniela], Tracés, $\mathrm{n}^{\circ} 16$, numéro hors-série, « traduire et introduire », p. 195-210. Disponible en ligne, https://journals.openedition.org/traces/6624 [lien valide en juin 2020].

MARELLA Maria Rosaria, 2017, « The commons as a legal concept », Law and Critique, vol. 28, p. 61-86.

MAROTTA Sergio, 2017, « Per una lettura sociologico-giuridica dei beni culturali come "beni comuni" ", in GEMMA Andrea, MASSARO Antonella, CORTESE Barbara \& BATTELLI Ettore (dir.), Patrimonio culturale. Profili giuridici e tecniche di tutela, Rome, Roma Tre-Press, coll. « L'unità del diritto ».

MATTEI Ugo, 2011, « Rendre inaliénables les biens communs », Le Monde diplomatique, vol. 693, $\mathrm{n}^{\circ}$ 12, p. 3. Disponible en ligne, https://www.monde-diplomatique.fr/2011/12/MATTEI/47058 [lien valide en juin 2020].

NAPOLI Paolo, 2014, « Indisponibilité, service public, usage. Trois concepts fondamentaux pour le “commun" et les "biens communs" », Tracés, n 27, « Penser avec le droit », p. 211-233. Disponible en ligne, https://journals.openedition.org/traces/6139 [lien valide en juin 2020].

ORSI Fabienne, 2014, « Réhabiliter la propriété comme bundle of rights : des origines à Elinor Ostrom, et au-delà ? », Revue internationale de droit économique, t. 28, p. 371-385. Disponible en ligne, https://www.cairn.info/revue-internationale-de-droit-economique-2014-3-page-371.htm [lien valide en juin 2020].

OST François, 1995a, La Nature hors la loi. L'écologie à l'épreuve du droit, Paris, La Découverte, coll. « Textes à l'appui », série « Écologie et société ».

OST François, 1995b, «Le milieu, un objet hybride qui déjoue la distinction public-privé », in CHEVALLIER Jacques (dir.), Public-privé, textes issu d'un séminaire de formation doctorale (Centre universitaire de recherches administratives et politiques de Picardie, 1993-1994), Paris, PUF, coll. « Publications du Centre universitaire de recherches administratives et politiques de Picardie ", p. 97 sq.

POUND Roscoe, 1959, "The socialization of law », Jurisprudence, vol. 2, The End of law, Saint Paul (Minnesota), West Publishing Company, p. 428 sq.

PUGLIATTI Salvatore, 1964, « La proprietà e le proprietà », in La Proprietà nel nuovo diritto, Milan, Giuffrè. 
ROCHFELD Judith, 2017, « Communauté négative, positive, diffuse » [notice], in CORNU Marie, ORSI Fabienne \& ROCHFELD Judith (dir.), Dictionnaire des biens communs, Paris, PUF, coll.

«Quadrige », p. 241-246.

RODOTÀ Stefano, 2016, « Vers les biens communs. Souveraineté et propriété au $\mathrm{XXI}^{\mathrm{e}}$ siècle » [in dossier « L'Italie des biens communs » dirigé par CHARBONNIER Pierre \& FESTA Daniela], Tracés, $\mathrm{n}^{\circ} 16$, numéro hors-série, « traduire et introduire », p. 212-232. Disponible en ligne, https://journals.openedition.org/traces/6632 [lien valide en juin 2020].

RODOTÀ Stefano, 2013, Il Terribile Diritto, studi sulla proprietà private e i beni comuni, Bologne, Il Mulino.

RODOTÀ Stefano, 2012, « Una democrazia “continua”? », Parole-chiave, nº 47, p. 1-5.

SANDULLI Aldo M., 1967, « La tutela del paesaggio nella Costituzione », RGE, n 2, p. 69 sq.

SASSEN Saskia, 2003, « Globalization or denationalization? », Review of international political economy, vol. 10, $\mathrm{n}^{\circ} 1$, p. 1-22.

SATTA Gino, 2013, « Patrimonio culturale », Parole-chiave, $n^{\circ}$ 49, p. 1-18.

SAUVÊTRE Pierre, 2016, « Les politiques du commun dans l'Europe du Sud (Grèce, Italie, Espagne). Pratiques citoyennes et restructuration du champ politique », Actuel Marx, $n^{\circ}$ 59, vol. 1 , p. 123-138.

SETTIS Salvatore, 2002, Italia SpA. L'assalto al patrimonio culturale, Turin, Einaudi, coll. « Struzzi ».

\section{NOTES}

1. L'expression, « assaut contre le patrimoine culturel », est de Salvatore Settis (2002), historien de l'art et directeur émérite de la Scuola Normale di Pisa qui a été un des acteurs les plus actifs dans la défense des biens culturels en tant que biens communs.

2. Voir en particulier l'interprétation d'inclusivité proposée par Séverine Dusollier (2015: en part. 104, $110 ; 2013: 258-281)$ et conjointement Judith Rochfeld (2017: 983-987). Pour des références incontournables, voir Crawford B. MacPherson (1962) et dans le même sens Stefano Rodotà (2013).

3. «La propriété est publique ou privée. Les biens économiques appartiennent à l'État, à des organismes ou à des particuliers. / La propriété privée est reconnue et garantie par la loi qui en détermine les modes d'acquisition, de jouissance ainsi que les limites afin d'en assurer la fonction sociale et de la rendre accessible à tous. / La propriété privée peut être expropriée, dans les cas prévus par la loi et sous réserve d'indemnisation, pour des motifs d'intérêt général. / La loi fixe les règles et les limites de la succession légale et testamentaire ainsi que les droits de l'État sur les héritages " (Constitution italienne, art. 42).

4. Sur l'héritage de Léon Duguit et sur son actualisation, voir en particulier Fabienne Orsi (2014: 371-385) et Thomas Boccon-Gibod (2014 : 285).

5. Voir Roscoe Pound (1959: 428), qui reprend l'expression de Jacques Charmont (1903: 380).

6. Pour une lecture incontournable, voir Duncan Kennedy (2006).

7. Voir le deuxième alinéa de l'art. 42 de la Constitution, dans la note 3 ci-dessus.

8. Je fais référence ici à l'éminente interprétation de Piero Calamandrei (1955 : 212).

9. «Dans des buts d'utilité générale, la loi peut réserver originairement ou transférer, par l'expropriation et sous réserve d'indemnisation, à l'État, à des établissements publics ou à des communautés de travailleurs ou d'usagers, des entreprises ou des catégories d'entreprises déterminées qui concernent des services publics essentiels ou des sources d'énergie ou des 
situations de monopole et qui ont un caractère d'intérêt général supérieur " (Constitution italienne, art. 43).

10. Les formules «biens culturels » ou «patrimoine culturel » sont plutôt récentes en Italie. L'expression «biens culturels» entre dans le vocabulaire officiel d'abord par le biais de l'institution du ministère des Biens culturels (1975) et ensuite avec l'actuel Codice per la tutela dei beni culturali e del paesaggio (d. lgs. 22 maggio 2004, $\mathrm{n}^{\circ} 42$ ) - qui reprend termes et approches d'une Commission parlementaire (1964-1967) instituée pour orienter la réforme de la discipline des choses d'intérêt artistique et pour fournir des principes organiques pour ce secteur. Cette Commission dite «Franceschini» (du nom de l'homme politique qui la préside) parlera de «biens culturels» comme «témoignages matériels ayant valeur de civilité " surlignant ainsi la double dimension d'héritage et de transmission du patrimoine culturel.

11. Pour une analyse approfondie, voir Antonio Bartolini (2013).

12. Pour une introduction aux concepts majeurs de cette école d'études territoriales, voir Alberto Magnaghi (2014).

13. Voir en particulier Lucio Gambi (1975 : 34-37).

14. J'utilise ici cette expression pour traduire librement « accadimenti emozionali », formule forgée par Massimo Severo Giannini (1976: 13).

15. Avec l'approbation de la Convention pour la sauvegarde du patrimoine culturel immatériel en 2003 (Satta 2013 : 1-18).

16. Cass., sez. un., 14 février 2011, n 3665, in Giust. civ., 2011, I, 595. En 2014, la CEDH appelle à se prononcer sur la question des Vallées de Pêches de la Lagune vénitienne reconnaissait une violation de l'art. 1, de la Convention internationale des droits de l'homme, qui considère le droit de propriété en tant que droit fondamental. La Cour européenne ne conteste pas toutefois les arguments de la Cour italienne et confirme qu'elle est le meilleur acteur pour décider des fonctions et des utilités publiques d'un bien au sein de son système. Cependant, la Cour européenne considère qu'en raison de la présence de nombreux éléments documentaires et preuves circonstancielles qui auraient pu faire présumer l'existence d'une titularité de la société recourant, l'État italien n'aurait pas garanti un balancement équitable des intérêts en refusant toute indemnité à la société.

17. Traduction libre.

18. Commission sur les biens publics créée en 2007 par le ministère de la Justice afin d'élaborer un projet de loi délégué pour reformer le chapitre du Code civil consacré à la discipline générale des biens. La Commission rassemblant plusieurs juristes était présidée par Stefano Rodotà, professeur émérite de droit civil et éminent homme politique, prendra donc le nom de ce dernier qui en sera ainsi le grand inspirateur et défenseur. Le rapport de la Commission a été remis en février 2008 et jamais discuté à cause de la dissolution prématurée du gouvernement qui l'avait constituée. Néanmoins, les contenus du rapport ont reçu une très grande diffusion et un consensus général parmi les juristes, les intellectuels et les organisations sociales en déclenchant un véritable mouvement des communs en Italie et promouvant l'important referendum de 2011 contre la privatisation de l'eau et des autres services publics locaux.

19. Dans ce sens, voir les lectures émérites de Vincenzo Cerulli Irelli (1994: 50) et d'Antonio Gambaro $(2012: 368)$.

20. En 1571, la loi du Grand-Duché de Toscane interdit aux propriétaires des immeubles d'altérer les signes des constructeurs et des fondateurs.

21. Stefano Rodotà $(2013 ; 2016)$ citant Crawford B. MacPherson (1962).

22. Massimo Severo Giannini (1976: 31) et Antonio Bartolini (2013 : 99).

23. SS.UU. Cass. cit. supra, voir note 19.

24. Je fais évidemment référence aux écrits séminaux de Hohfeld 1913 et 1917.

25. Voir Massimo Severo Giannini (1976), et dans le même sens Vincenzo Cerulli Irelli (1994) qui selon le même raisonnement qualifie les biens culturels de res publicae. 
26. On peut cependant citer l'analyse intéressante de Saleilles autour de la notion de propriété artistique où il décompose les droits de l'État, les droits du propriétaire corporel et le droit supérieur de l'auteur de l'œuvre (Cornu $2014:$ 185-196).

27. Selon la lecture de Pierre Calata (1966 : 186) citée par Ost1995b : 101.

28. Pour une interprétation complète et systématique de ces droits, voir Vincenzo Cerulli Irelli (1983).

29. Pour une introduction aux intérêts diffus dans la tradition italienne, voir Chiara Agiolini (2017) et pour leur interprétation au sein du droit portugais : Aragão \& Carvalho (2017 : 42-48).

30. Pour une récente relecture éclairante de la pensée de Salvatore Pugliatti, voir Anne Di Robilant (2013 : en part. 905).

31. Pour ce concept et pour une lecture approfondie des évolutions du patrimoine et son croisement avec les biens communs, voir Sergio Marotta (2017).

32. Je reprends ici la formule vigoureuse de Salvatore Settis (2002).

33. Grossi «Droits fonciers collectifs » et, du même auteur : "Droits civiques d'usage » (Cornu et al. 2017).

34. Commission mieux connue sous le nom « Commission Rodotà », voir supra note 16.

35. Sur le caractère paradigmatique de cette connexion dans le cadre des mouvements du commun, voir Dardot \& Laval (2014: 595).

36. Il s'agit du « claim » qui rassemblera le mouvement pour le referendum d'initiative populaire contre la privatisation des services publics. Il se déroulera en juin 2011 avec une victoire des voix contraires à une très large majorité.

37. Pour un approfondissement, je me permets ici de renvoyer à Festa (2017a).

38. «Les fleuves, les torrents et leurs sources; les lacs et les autres milieux aquatiques; l'air, les parcs selon la définition de la loi, les forêts et les bois, les zones de montagne de haute altitude, les glaciers et les neiges éternelles; les littoraux et les étendues littorales déclarées réserve naturelle ; la faune et la flore protégées ; les biens archéologiques, culturels, environnementaux et les autres zones paysagères protégées sont des biens communs parmi d'autres » (art. 1, let.c) Projet de loi déléguée proposé).

39. Stefano Rodotà dans Festa \& Charbonnier (2016:215).

40. Ladite école de Bloomington se forme autour de Elinor et Vincent Ostrom et produit un riche ensemble d'analyses concernant les facteurs clefs de succès de l'auto-organisation dans l'accès et la gestion de ressources.

41. Pour un aperçu en langue français, voir Rodotà (2016), Mattei (2011:3) et Lucarelli (2019).

42. La fondation sera légalement constituée auprès d'un notaire mais elle ne recevra pas la reconnaissance par le préfet car le statut envisage de fixer le siège dans le théâtre même sans pouvoir revendiquer aucun titre légitime sur l'immeuble toujours en état d'occupation. Les occupants abandonnent le théâtre à la suite de fortes pressions politiques, après trois années d'intenses activités culturelles et expérimentations politiques qui ont donné vie à une des expériences de commoning les plus notoires et appréciées en Europe. Le théâtre demeure depuis lors fermé et en attente de travaux de restauration prévus depuis longtemps mais jamais réalisés, le département responsable de la sauvegarde des biens culturels a par ailleurs établi que l'occupation n'a produit aucun endommagement du bâtiment classé et a su le préserver et l'entretenir à l'identique.

43. Selon Salvatore Pugliatti (1964 : 218) et selon Sent Cass. cit.

44. Pour une lecture critique de ces règlements, je me permets de renvoyer à Festa (2016).

45. Convention-cadre du Conseil de l'Europe sur la valeur du patrimoine culturel pour la société, signée à Faro en 2005.

46. Selon l'art. 11, troisième alinéa de la charte de Pérouse disponible en ligne: https:// www.comune.perugia.it/resources/regolamenti/02.11_CollaborazioneBeniComuniUrbani.pdf [lien valide en mars 2021]. 
47. Pour un aperçu des différents modèles aux Etats-Unis, voir Foster (2011). Pour une lecture d'ensemble, voir Foster \& Iaione (2016: 281-349).

48. Plus d'information sur le site : http://www.burenvandeabdij.be/ [lien valide en juin 2020].

49. Voir de manière similaire le modèle de jardins partagés régis par le programme Main verte de la Ville de Paris.

50. Elle reçoit en 2009 The Sveriges Riksbank prize in economic sciences in memory of Alfred Nobel.

51. Pour un approfondissement au sujet de cette notion de lieux de la production contemporaine à considérer biens communs en tant que patrimoine de la production immatérielle, je me permets de renvoyer à Festa (2017b).

52. Dans ce sens, voir Pierre Sauvêtre (2016: 123-138), qui reprend la réflexion de Pierre Dardot au sujet de la relativisation du rôle de l'État.

53. La notion est élaborée par Stefano Rodotà (2012) et articule efficacement biens communs de la connaissance et de l'information via Intenet et biens communs matériels.

54. Voir supra, note 1.

55. Je reprends la définition éclairante de Morris R. Cohen (1927).

56. Dans le même sens, voir également Marella (2017) et Ferrari (2016).

57. Sur cette notion avancée par Salvatore Pugliatti (1964:159), voir Di Robilant (2013: 905).

58. SS. UU. Cass. cit. supra, note 16 .

\section{RÉSUMÉS}

Pouvons-nous considérer les biens culturels en tant que biens communs ou communs? Peut-on interpréter la relation à ces biens sous l'angle de l'inclusivité et non pas de l'exclusivisme propriétaire et dans quel sens?

L'article tente une réponse à ces questions en montrant d'abord, comment dans la tradition juridique italienne la notion des biens culturels s'est forgée, au fil du dernier siècle, en relation constante avec une conception de propriété en tant que droit fonctionnalisé. Les biens culturels ont ainsi su représenter conjointement avec les biens paysagers et environnementaux, un modèle de propriété divise et d'affectation collective. Réinterrogés par les pratiques de commoning et par la théorie de biens communs, ils doivent aujourd'hui être appréhender sous l'angle de l'accès effectif aux utilités engendrées par la chose et celui de la participation active afin de garantir l'épanouissement de la personne dans sa dimension individuelle et collective.

Can we consider cultural goods as commons or common goods? Can the relationship to these goods be interpreted as inclusive instead that under the paradigm of exclusivity?

The article attempts to answer these questions by first showing how, in the Italian legal tradition, the notion of cultural heritage goods has been forged over the past century in constant relation to a conception of property as a functionalized right. Cultural goods have thus been able to represent, together with landscape and environmental properties, a model of dismembered property with a collective dedication. Revised by practices of commoning and the theory of common goods, they must now be understood from the double perspective of an effective access to the utilities generated by the thing and of an active participation in order to guarantee the flourishing of the person in his individual and collective dimension. 
cultural goods, cultural heritage, landscape, commons, common goods, dismembered property, collective dedication

INDEX

Mots-clés : biens culturels, patrimoine, paysage, communs, biens communs, propriété divise, affectation collective

\section{AUTEUR}

DANIELA FESTA

Department of Sociology, Columbia University, Visiting scholar festadaniela@gmail.com 